\title{
Quasi-transversal saddle-node bifurcation on surfaces
}

\author{
J. BELOQUI† \\ Instituto de Matemática e Estatística, Universidade de São Paulo, C.P. 20570, \\ CEP 01498, S. Paulo-SP, Brasil \\ AND \\ M. J. PACIFICO† \\ Instituto de Matemática, Universidade Federal do Rio de Janeiro, C.P. 68530, \\ CEP 21910, Rio de Janeiro-RJ, Brasil
}

(Received 28 June 1988 and revised 9 March 1989)

\begin{abstract}
In this paper we give a complete set of invariants (moduli) for mild and strong semilocal equivalence for certain two parameter families of diffeomorphisms on surfaces. These families exhibit a quasi-transversal saddle-connection between a saddle-node and a hyperbolic periodic point.
\end{abstract}

\section{Introduction}

Generically diffeomorphisms with simple recurrence are Morse-Smale and therefore structurally stable [9]. It is conjectured that generically one parameter families of diffeomorphisms, starting at a Morse-Smale one, first bifurcate either by the loss of hyperbolicity of a periodic orbit or by the appearance of a nontransversal saddle-connection [10]. Such one-parameter families may not be stable, but in several relevant cases the classes of equivalence may be parametrized by finitely many real invariants (moduli) as introduced in [8]. To be more precise, let us recall some definitions and results.

Two smooth families of $C^{\infty}$ diffeomorphisms of $M^{2}, f_{\mu}$ and $\tilde{f}_{\mu}, \mu \in \mathbf{R}^{n}$, are mildly equivalent if there exists a reparametrization $\rho: \mathbf{R}^{n} \gg$ and a family $h_{\mu}: M \gg$ of homeomorphisms satisfying the equation

$$
f_{\mu} \circ h_{\mu}=h_{\mu} \circ \tilde{f}_{\rho(\mu)} \text {. }
$$

If in addition, $h_{\mu}$ varies continuously with $\mu$, we say that $f_{\mu}$ and $\tilde{f}_{\mu}$ are strongly equivalent or simply equivalent. We say that a family $f_{\mu}$ is (strongly) stable if it is equivalent to all nearby ones.

† Partially supported by CNPq-Brasil. This paper was prepared mainly at the Instituto de Matemática, Universidade Federal do Rio de Janeiro, to whom the first author acknowledges hospitality. 
A complete characterization of stable generic arcs $f_{\mu}$ of diffeomorphisms such that $f_{\mu}$ is Morse-Smale for $\mu<0$ and $f_{0}$ has simple recurrence was established in [11]. They proved in fact that one of the periodic orbits of $f_{0}$ must be nonhyperbolic and that this orbit is either a saddle-node or a flip. Also, in [11], it is proved that arcs going through either a saddle-node or a flip orbit are strongly stable near these orbits. But arcs passing through a Hopf orbit are not even mildly stable due to the appearance of invariant circles where the restriction of the diffeomorphisms have irrational rotation number.

Now, in the same context, if the diffeomorphism at the first point of bifurcation has simple recurrence and all periodic orbits are hyperbolic then their unstable and stable manifolds meet transversally except along one orbit. In this case the arc is not even mildly stable. For instance, let $f$ be a diffeomorphism of a surface which exhibits two hyperbolic fixed points $p, q$ such that $W^{u}(p)$ meets $W^{s}(q)$ along a unique orbit of quasi-transversal intersection (parabolic contact) and let $g$ be near $f$ so that it also exhibits a unique orbit of quasi-transversal intersection between $W^{u}(\tilde{q})$ and $W^{s}(\tilde{p})$, where $\tilde{q}$ and $\tilde{p}$ are fixed points for $g$ near $q$ and $p$. For $f$ and $g$ to be equivalent near the closure of this orbit it is necessary and sufficient that

$$
\lambda=\frac{\log \beta^{u}(q)}{\log \beta^{s}(p)}=\frac{\log \tilde{\beta}^{u}(\tilde{q})}{\log \tilde{\beta}^{s}(\tilde{p})}=\tilde{\lambda},
$$

where $\beta^{\sigma}(\gamma)$ (resp. $\tilde{\beta}^{\sigma}(\tilde{\gamma})$ ) is the eigenvalue associated to $W^{\sigma}(\gamma)$ (resp. $W^{\sigma}(\tilde{\gamma})$ ), $\sigma=s, u ; \gamma=p, q$. Thus there is a differentiable real invariant which is preserved under topological equivalence. In this context we call $\lambda$ a modulus for the equivalence. In [14] it is proved that for a generic one parameter family of diffeomorphisms going through a quasi-transversal bifurcation, there are two moduli for strong equivalence; namely $\beta^{s}(q)$ and $\beta^{u}(p)$. For mild equivalence there is one modulus which is $\lambda$.

For one parameter families of vector fields, a complete set of topological invariants near quasi-transversal saddle-connections was established in [14], after the initial contributions of [13] and [1].

For generic two-parameter families of diffeomorphisms, even the local study near a codimension-two fixed point is not yet complete. Nevertheless, there are important results like in [4], where the unfolding of a codimension-two Hopf bifurcation is analysed. The case of two eigenvalues equal to one and a nontrivial nilpotent part is essentially open. However, the corresponding unfolding and local equivalence for vector fields is known [2, 13]. More recently, in [7], codimension-two saddlenodes, flips and reflections (eigenvalues 1 and -1 ) were also treated, and global stability involving such cases has also been analysed. Bifurcations in two-parameter families of diffeomorphisms with simple recurrence include also cubic tangencies and cascade of tangencies. This was studied in [6] where the authors characterize surface diffeomorphisms having simple hyperbolic recurrence and a finite moduli of stability.

However, diffeomorphisms in generic two-parameter families may also have simultaneously a nontransversal saddle-connection and a nonhyperbolic periodic 
orbit, which may be a flip, a Hopf orbit or a saddle-node. Here we will study a two-parameter family of diffeomorphisms for which $f_{\mu_{0}}$ exhibits a quasitransversal saddle-connection between a saddle-node and a hyperbolic fixed (periodic) point.

Let us describe the families we will deal with.

Let Diff ${ }^{\infty}\left(M^{2}\right)$ be the space of $C^{\infty}$ diffeomorphisms endowed with the $C^{\infty}$ Whitney topology and let $\operatorname{Diff}_{2}^{\infty}\left(M^{2}\right)$ be the space of mappings $f: I \times I \rightarrow \operatorname{Diff}^{\infty}\left(M^{2}\right), I=$ $[-1,1]$ with the usual $C^{\infty}$ topology. For short we write $f_{\mu}$ instead of $f(\mu), \mu \in I \times I$.

Suppose that $f_{\mu}$ is a 2-parameter family of diffeomorphisms such that at $\mu=0$, $f_{o}$ exhibits:

(a) a hyperbolic saddle fixed point $p=p_{o}, C^{2}$ linearizable, with contracting eigenvalue $\alpha \in \mathbf{R}$

(b) a saddle-node fixed point $q=q_{o}$ such that $W^{u}(p)$ intersects $W^{s}(q)$ quasi transversally along a unique orbit $\gamma$. Here there are two possibilities:

$\left(b_{1}\right)$ either $W^{s}(q)$ is a submanifold with boundary $W^{s s}(q)$ and $W^{u}(q)$ intersects $W^{s s}(q)$ or

$\left(b_{2}\right) W^{u}(q)$ is a submanifold with boundary $W^{u u}(q)$ and $W^{u}(p)$ intersects $W^{s}(q)$.

In any case we call $\beta$ the expanding eigenvalue at $q$.

The set of diffeomorphisms satisfying $\left(b_{1}\right)$ (resp. $\left(b_{2}\right)$ ) above is denoted by $S N Q T 1$ (resp. SNQT2). We denote $S N Q T$ the union of $S N Q T 1$ and $S N Q T 2$.

We shall prove that $S N Q T$ is a codimension-two manifold in $\operatorname{Diff}^{\infty}\left(M^{2}\right)$. Hence, a generic two parameter family $f_{\mu}$ meets $S N Q T$ transversally at $f_{o}$, which we call the central bifurcation for $f_{\mu}$. The set of 2-parameter families for which $f_{o} \in S N Q T 1$ (resp. $S N Q T 2$ ) is denoted by $S N Q T 1^{\prime}$ (resp. $S N Q T 2^{\prime}$ ). We denote by $S N Q T^{\prime}$ the union of $S N Q T 1^{\prime}$ and $S N Q T 2^{\prime}$.
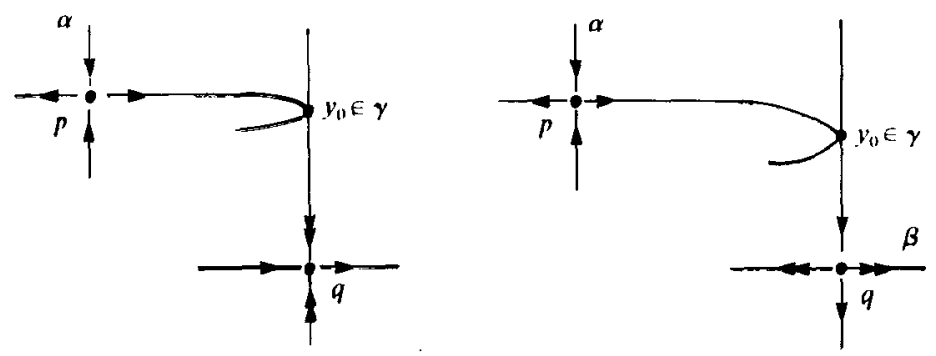

Let us now give the semilocal versions of the notions of equivalence.

A semi-local equivalence between $f$ and $\tilde{f}$ in $S N Q T$ is an equivalence defined from a neighbourhood of the closure of $\tilde{\gamma}$. We will prove:

THEOREM A. (1) Let $f, \tilde{f}$ be two $C^{r}$ nearby diffeomorphisms in SNQT1. Then they are semilocally equivalent if and only if $\alpha=\tilde{\alpha}$. (2) Let $f, \tilde{f}$ be two $C^{r}$ nearby diffeomorphisms in SNQT2. Then they are semilocally equivalent if and only if

$$
\frac{\log \beta}{\log \alpha}=\frac{\log \tilde{\beta}}{\log \tilde{\alpha}} .
$$

Thus there is one modulus of stability for diffeomorphisms in SNQT. 
THEOREM B. Two nearby families $f_{\mu}$ and $\tilde{f}_{\mu}$ in $S N Q T^{\prime}$ are mildly equivalent if and only if their central bifurcations are equivalent.

Again, there is one modulus of mild stability for families in $S N Q T^{\prime}$.

Before we state the next theorem, we describe some invariants for strong stability.

First Case: $f_{\mu} \in S N Q T 1^{\prime}$. Here, the set of $f_{\mu}$ 's which exhibits a tangency between $W^{u}(p)$ and $W^{s s}(q)$ is a curve in the space of parameters, along which we need $\alpha=\tilde{\alpha}$ and $\beta=\tilde{\beta}$ [14]. For $\beta$ small enough, we may use it as a parameter and describe the modulus as a function $\alpha=\alpha(\beta)$.

Second Case: $f_{\mu} \in S N Q T 2^{\prime}$. The set of $f_{\mu}$ which exhibits a tangency between $W^{u}(p)$ and $W^{c}(q)$ will be a curve along which we must have $\alpha=\tilde{\alpha}$ and $\beta=\tilde{\beta}$ [14]. But now some other moduli arise at the saddle-nodes. Indeed, in [11] it is proved that if $f_{\mu}$ is a diffeomorphism which has a saddle-node $q_{\mu}$, then $\left.f_{\mu}\right|_{W^{c}\left(q_{\mu}\right)}$ is the time one map of a vector field $X_{\mu}$, called an adapted vector field. Moreover, if $h_{\mu}$ is a conjugacy between two such diffeomorphisms, then $\left.h_{\mu}\right|_{w^{c}\left(q_{\mu}\right)}$ is also a conjugacy between the corresponding adapted vector fields. This conjugacy is differentiable at $W^{c}\left(q_{\mu}\right) \backslash\left\{q_{\mu}\right\}$.

The moduli are related to the rigidity of $\left.h_{\mu}\right|_{W^{c}\left(q_{\mu}\right)}$ and arise along the set of saddle-nodes for which $W_{\mu}^{u}(p) \cap W_{\mu}^{c}(q) \neq \Phi$. Performing a suitable reparametrization this set may be represented by the semi axis $\left\{\mu_{2} \geq 0, \mu_{1}=0\right\}$. Let us describe the invariants:

(1) $\alpha_{\mu}=\tilde{\alpha}_{\mu}$ due to the differentiability of $h_{\mu} \mid w_{\mu}$.

Let $N$ be a fundamental neighbourhood for $W_{\mu}^{u}(p), y_{o} \in N$, and $\left\{a_{\mu}, b_{\mu}\right\}=W_{\mu}^{u}(p) \cap$ $W_{\mu}^{c}(q) \cap N$. Then

(2) $t\left(0, \mu_{2}\right)=\tilde{t}\left(0, \tilde{\mu}_{2}\right)$ where $X_{t}\left(a_{\mu}\right)=b_{\mu}, \tilde{X}_{\tilde{i}}\left(\tilde{a}_{\mu}\right)=\tilde{b}_{\mu}$,

(3) the normal derivatives at $a_{\mu}, b_{\mu}$ are equal, i.e., $k\left(a_{\mu}\right)=k\left(b_{\mu}\right)$, as a consequence of the differentiability of $\left.h\right|_{w_{\mu}}$.

Through a convenient reparametrization we can get rid of one of these invariants. So if we choose the reparametrization given implicitly by $t\left(0, \mu_{2}\right)=\tilde{t}\left(0, \tilde{\mu}_{2}\right)$ we may express the resulting moduli as

We finally state

$$
\left\{\begin{array}{l}
\alpha_{\mu}=\tilde{\alpha}_{\mu} \text { and } \\
k\left(a_{\mu}\right)=k\left(b_{\mu}\right)
\end{array}\right.
$$

THEOREM C. For generic families in $S N Q T^{\prime}$ the complete set of moduli for strong equivalence is

(1) $\alpha(\beta)=\tilde{\alpha}(\beta)$ for $f_{\mu}, \tilde{f}_{\mu} \in S N Q T 1^{\prime}$

(2) $\alpha(\beta)=\tilde{\alpha}(\beta)$ along the curves of tangencies and

$$
\left\{\begin{array}{l}
\alpha_{\mu}=\tilde{\alpha}_{\mu} \\
k\left(a_{\mu}\right)=k\left(b_{\mu}\right)
\end{array}\right.
$$

along the curves of saddle-nodes for $f_{\mu}, \tilde{f}_{\mu} \in S N Q T 2 '$ '.

Hence, the space of moduli is given by a space of germs of real functions, showing that for strong stability the moduli space is much richer than for mild equivalence. 
Nevertheless, they represent the full description of the equivalence classes of the bifurcating two-parameter families described above.

In higher dimensions, the same question can be posed. We observe that new moduli may appear due to the existence of a least contracting (resp. expanding) eigenvalue at the saddle-node. Another possible, and yet not studied bifurcation in higher dimension corresponds to a diffeomorphism with a quasi-transversal saddleconnection between hyperbolic points $p$ and $q$ where there are two least contracting eigenvalues at $p$ or two least expanding ones at $q$.

Acknowledgement. We are thankful to R. Labarca and J. Palis for helpful and stimulating conversations.

We are also thankful to the referee for good suggestions, including a simplification of one of our proofs.

\section{Bifurcation diagrams}

In this section we exhibit the bifurcation diagrams for $f_{\mu} \in S N Q T^{\prime}$.

We start by choosing appropriate coordinates and parameters to describe $f_{\mu}$. One of the parameters, $\lambda_{1}$, will be related to the loss of hyperbolicity and the other, $\lambda_{2}$, to the loss of transversality.

For $f_{(0,0)} \in S N Q T$ we may choose a domain $L$ where all diffeomorphisms near $f_{(0,0)}$ are $C^{2}$ linearizable in $L[12]$. Identify $L$ with a neighbourhood of $(0,0)$ in $\mathbf{R}^{2}$ so that we can write $g(x, y)=(\nu x, \alpha y), g$ near $f_{(0,0)}$. Let $W \subset W_{\mu}^{u}(p)$ be a small compact connected neighbourhood of $p$ in $W_{\mu}^{u}(p)$. We call the closure of $W \backslash f_{\mu}^{-1}(W)$ a fundamental domain $D_{\mu}$ for $W_{\mu}^{u}(p)$. Denote the local stable manifold of $p$ by $W_{\mu, \text { loc }}^{s}(p)$. Any neighbourhood of $D_{\mu}$ that is disjoint from $W_{\mu, \text { loc }}^{s}(p)$ is called a fundamental neighbourhood for $W_{\mu}^{u}(p)$. We choose a fixed fundamental neighbourhood $N$ containing $D_{\mu}$ for all $\mu, N \cap f_{\mu}^{-2}(N)=\Phi$. Note that both $D_{\mu}$ and $N$ have two connected components.

For a family $f_{\mu} \in S N Q T^{\prime}$, there exists $W_{\mu}^{c}$ a one dimensional $C^{k}$ invariant central manifold, which depends continuously on $\mu$ [11]. Also, the unfolding of $\left.f_{\mu}\right|_{w_{\mu}^{c}}$ is

$$
f_{\mu}(x)=\lambda_{1}(\mu)+x+x^{2}+x^{2} \sigma(x, \mu), \quad \mu \in U,
$$

where $U$ is a neighbourhood of $\mu=0$ in $\mathbf{R}^{2}$.

Observe that $\lambda_{1}=\lambda_{1}(\mu) \in C^{2}$ and $f_{\mu}$ exhibits a saddle-node fixed point only for $\lambda_{1}=0$. From this expression we determine formulae for the hyperbolic fixed points:

$$
f_{\mu}(q)=q \text { if and only if } \lambda_{1}+q^{2}+q^{2} \sigma(q, \mu)=0 .
$$

So that $q \cong \pm \sqrt{\left|\lambda_{1}\right|}$ for $\lambda_{1} \leq 0$, that is $q / \pm \sqrt{\left|\lambda_{1}\right|} \rightarrow 1$ as $\lambda_{1} \rightarrow 0$. Call $q_{1} \cong-\sqrt{\left|\lambda_{1}\right|}, q_{2} \cong \sqrt{\left|\lambda_{1}\right|}$. Since $(\partial / \partial x) f_{\mu}\left(q_{1}\right)<1$ and $(\partial / \partial x) f_{\mu}\left(q_{2}\right)>1, q_{1}$ is a saddle fixed point and $q_{2}$ is a sink for $f_{\mu}$.

Note that the distance $d\left(q_{1}, q_{2}\right) \cong 2 \sqrt{\left|\lambda_{1}\right|}$.

Now we choose $\lambda_{2}$ for $f_{(0,0)} \in S N Q T 1$.

Let $F_{\mu}\left(q_{o}\right)$ be a $C^{2}$ curve in $M^{2}$ transversal to $W_{\mu}^{c}$, continuously depending on $\mu$, such that $F_{o}\left(q_{o}\right)=W_{o}^{s s}\left(q_{o}\right)$. Take $y_{o}$ a point in the orbit of tangency in the domain of linearization around $p$. When no confusion is possible we still denote by $N$ 
the connected component of the fundamental neighbourhood for $W_{\mu}^{u}(p)$ which contains $y_{o}$.

Define $\left|\lambda_{2}\right|=d\left(W_{\mu}^{u}(p) \cap N, F_{\mu}\left(q_{o}\right) \cap N\right)$ and $\lambda_{2}>0$ if and only if $W_{\mu}^{u}(p)$ and $W_{\mu}^{s s}\left(q_{1}\right)$ belong to the same connected component of $N \backslash F_{\mu}\left(q_{o}\right)$. Observe that $\lambda_{2}$ depends differentiably on $\mu \in U$ and the distance $d\left(F_{\mu}\left(q_{o}\right) \cap N, W_{\mu}^{s s}\left(q_{1}\right) \cap N\right)$ is proportional to $\sqrt{\left|\lambda_{1}\right|} \cong d\left(F_{\mu}\left(q_{o}\right) \cap W_{\mu}^{c}, q_{1}\right)$.

For this choice of parameters we define $\mathscr{A}_{1} \subset S N Q T 1^{\prime}$ as the set of families for which the jacobian matrix $J\left(\lambda_{1}, \lambda_{2}\right)(\mu)$ at $\mu=(0,0)$ is nonsingular. For technical reasons we also assume that $\left(\partial \beta / \partial \lambda_{2}\right)(0,0) \neq 0$.

By the Inverse Function Theorem, in a neighbourhood of $(0,0)$ we can express $\mu=\left(\mu_{1}, \mu_{2}\right)$ with

$$
\begin{aligned}
& \mu_{1}=\mu_{2}\left(\lambda_{1}, \lambda_{2}\right) \\
& \mu_{2}=\mu_{2}\left(\lambda_{1}, \lambda_{2}\right) .
\end{aligned}
$$

From now on we consider $f_{\mu}$ parametrized by $\left(\lambda_{1}, \lambda_{2}\right)$, i.e., $\mu_{1}=\lambda_{1}, \mu_{2}=\lambda_{2}$.

Due to this choice of $\mu_{1}$ and $\mu_{2}$ we easily derive the bifurcation diagram for $f_{\mu} \in \mathscr{A}_{1}$. Since we are only concerned with semiglobal properties, we fix a connected neighbourhood $V$ of $p, q$ and the orbit of $y_{o}$ by $f_{(0,0)}$.

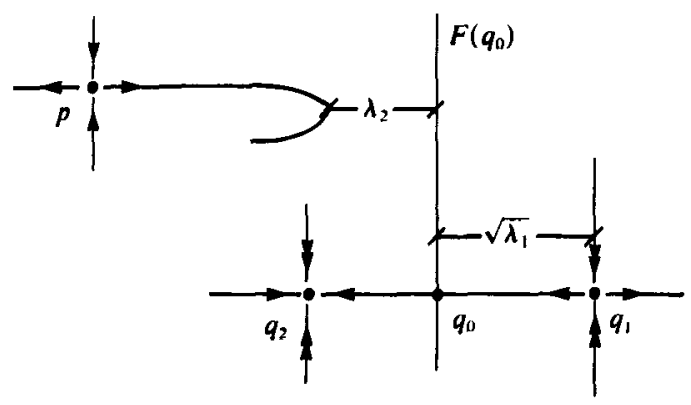

The set of saddle-nodes corresponds to $\mu_{1}=0$ and tangencies between invariant manifolds associated to hyperbolic fixed points are, up to a constant factor given by

$$
\mu_{2}=\xi\left(\mu_{1}\right) \cong-\sqrt{\left|\mu_{1}\right|}, \quad \mu_{1} \leq 0 .
$$

Indeed, the distance between $F_{\mu}\left(q_{o}\right)$ and $W_{\mu}^{s}\left(q_{1}\right)$ is proportional to $\sqrt{\left|\mu_{1}\right|}$, as we remarked above.

Finally, for every other value of $\mu=\left(\mu_{1}, \mu_{2}\right)$, the restriction of $f_{\mu}$ to $V$ is a Morse-Smale diffeomorphism.

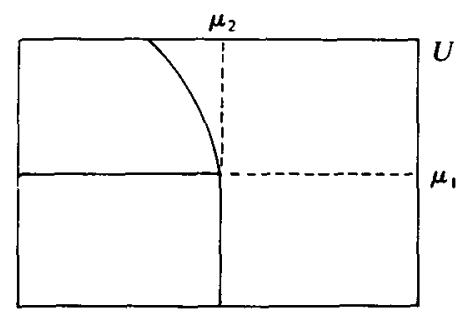


Now we turn our attention to families $f_{\mu}$ in $S N Q T 2^{\prime}$. Take $V$ a connected neighbourhood of $p, q$ and the orbit of $y_{o}$ by $f_{(0,0)}$ and let $N$ be a fundamental neighbourhood of $y_{o}, N \subset V$.

We choose $\lambda_{1}$ similarly. Note that here $q_{2}$ is a source.

For $\lambda_{2}$ we set

$$
\left|\lambda_{2}\right|=d\left(W_{\mu}^{u} \cap N, W_{\mu}^{c} \cap N\right)
$$

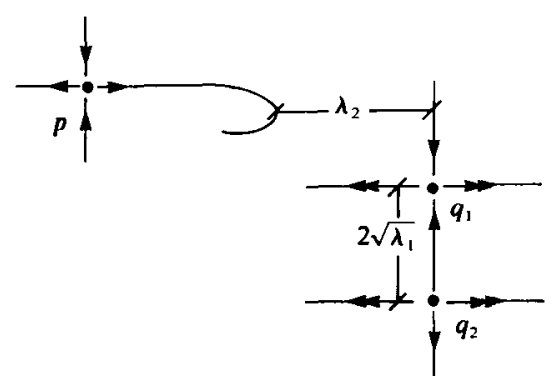

and $\lambda_{2} \geq 0$ if and only if $N \cap W_{\mu}^{u}(p) \cap W_{\mu}^{c} \neq \Phi$.

As before we consider $\mathscr{A}_{2}$ the set of families in $S N Q T 2^{\prime}$ for which $J\left(\lambda_{1}, \lambda_{2}\right)(0,0)$ is nonsingular and

$$
\frac{\partial}{\partial \lambda_{1}}\left(\frac{\log \beta}{\log \alpha}\right)(0,0) \neq 0 .
$$

Hence, the Inverse Function Theorem allows us to take $\mu_{1}=\lambda_{1}$ and $\mu_{2}=\lambda_{2}$. In this case the distinguished sets in the bifurcation diagram are:

$\mu_{1}=0$ which corresponds to the curve of saddle-nodes and

$\mu_{0}=0, \mu_{1} \leq 0$ which corresponds to the curve of tangencies.

For every other value of $\left(\mu_{1}, \mu_{2}\right)$, the restriction of $f_{\mu}$ to $V$ is a Morse-Smale diffeomorphism.

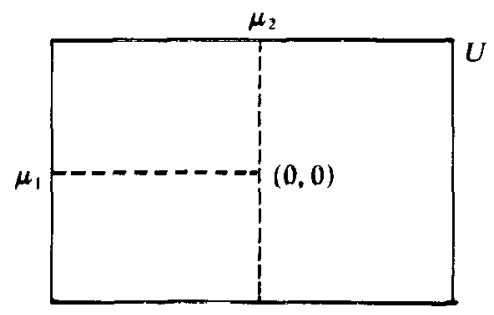

Two important consequences derive from our choice of $\mathscr{A}_{1}$ and $\mathscr{A}_{2}$ :

(1) $\mathscr{A}_{1}$ is open and dense in $S N Q T i^{\prime}, i=1,2$.

(2) each family $f_{\mu} \in \mathscr{A}_{i}, i=1,2$, intersects $S N Q T i$ at a unique point $f_{(0,0)}$, which we call the central bifurcation.

Let us describe the type of diffeomorphisms that lie in $f_{\mu}$ near $f_{(0,0)}$.

(i) $f_{\mu} \in \mathscr{A}_{1}, \quad \mu \in U \subset \mathbf{R}^{2}$.

From the bifurcation diagram we have that $U \backslash\left(\left\{\left(\mu_{1}, \xi\left(\mu_{1}\right)\right), \mu_{1} \geq 0\right\} \cup\left\{\mu_{1}=0\right\}\right)$ has three connected components $U_{i}, 1 \leq i \leq 3$. 
It is easy to see that for each $i$, all diffeomorphisms $f_{\mu}, \mu \in U_{i}$, are equivalent. Moreover,

(a) for $f_{\mu_{o}}, \mu_{o} \in U_{1}$, there are three fixed points $p, q_{1}, q_{2}$ in $V$, all of them hyperbolic and $W_{\mu}^{u}(p) \cap W_{\mu}^{s}\left(q_{1}\right)=\Phi$,

(b) for $f_{\mu_{o}}, \mu_{o} \in U_{2}$, there are three fixed points $p, q_{1}, q_{2}$ in $V$, all of them hyperbolic but $W_{\mu}^{u}(p)$ and $W_{\mu}^{s}\left(q_{1}\right)$ meet transversally,

(c) for $f_{\mu_{o}}, \mu_{o} \in U_{3}, p$ is the only fixed point in $V$.

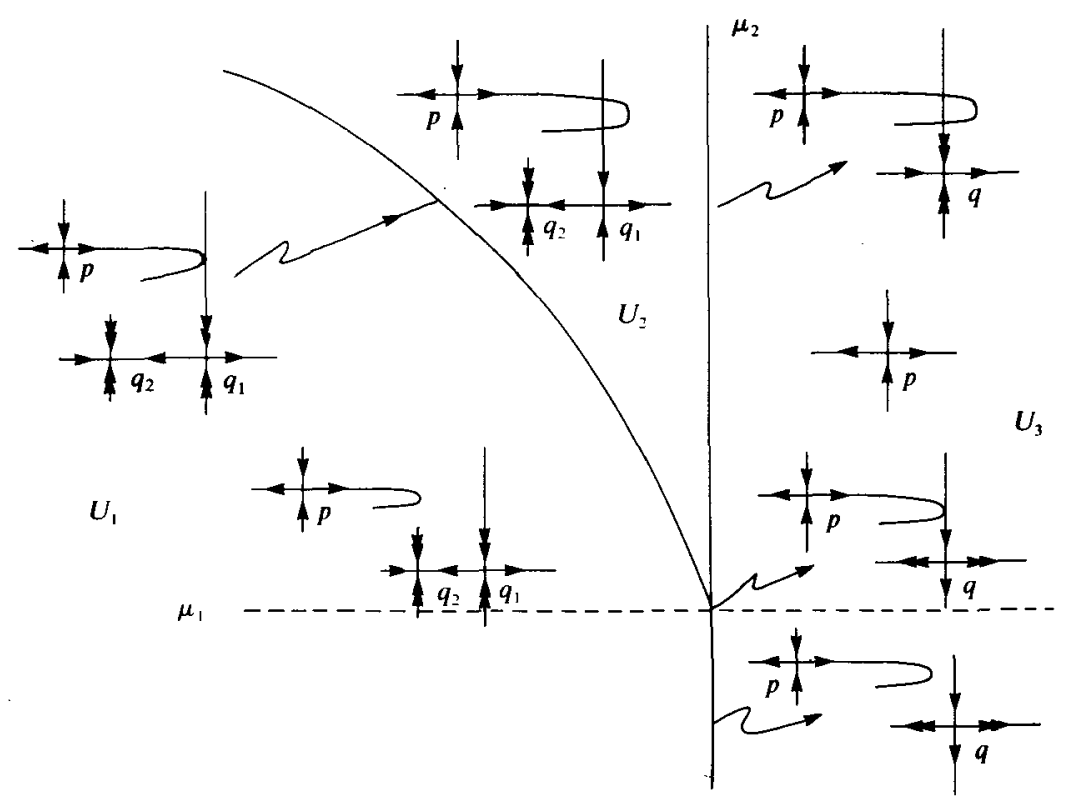

For values $\left(\mu_{1}, \xi\left(\mu_{1}\right)\right), W_{\mu}^{u}(p)$ and $W_{\mu}^{s s}\left(q_{1}\right)$ meet quasi-transversally, while $p$ and $q_{1}$ are hyperbolic. This gives rise to a modulus of equivalence [8], namely $\log \beta / \log \alpha$.

Along the curve of saddle-nodes, i.e., $\left\{\mu_{1}=0\right\}$, we have three classes of equivalence: one corresponding to $f_{\left(0, \mu_{2}\right)}, \mu_{2}>0$, another to $f_{\left(0, \mu_{2}\right)}, \mu_{2}<0$, and the last one to the central bifurcation.

Indeed, for $f_{\left(0, \mu_{2}\right)}, \mu_{2}>0, W^{u}(p)$ intersects $W^{s s}(q)$ transversally, whereas for $f_{\left(0, \mu_{2}\right)}, \mu_{2}<0, W^{u}(p)$ does not intersect $W^{s s}(q)$.

From [11] it follows that all $f_{\left(0, \mu_{2}\right)}, \mu_{2}>0$ (resp. $\mu_{2}<0$ ) determine the same class of topological equivalence.

Clearly, the central bifurcation $f_{(0,0)}$ is not equivalent to any other $f_{\mu}, \mu \neq(0,0)$. (ii) $f_{\mu} \in \mathscr{A}_{2}, \quad \mu \in U \subset \mathbf{R}^{2}$.

From the bifurcation diagram we deduce that $U \backslash\left(\left\{\mu_{1}=0\right\} \cup\left\{\mu_{2}=0, \mu_{1} \leq 0\right\}\right)$ has three connected components $U_{i}, 1 \leq i \leq 3$. All diffeomorphisms $f_{\mu}$, with $\mu$ in $U_{i}$, are equivalent. Furthermore,

(a) for $f_{\mu_{o}}, \mu_{o} \in U_{1}=\left\{\left(\mu_{1}, \mu_{2}\right) ; \mu_{1}<0, \mu_{2}<0\right\}$ there are three fixed points $p, q_{1}, q_{2}$ in $V$, all of them hyperbolic, and $W_{\mu}^{u}(p) \cap W_{\mu}^{s}\left(q_{1}\right)=\Phi$,

(b) for $f_{\mu_{o}}, \mu_{o} \in U_{2}=\left\{\left(\mu_{1}, \mu_{2}\right) ; \mu_{1}<0, \mu_{2}>0\right\}$ there are three fixed points $p, q_{1}, q_{2}$ in $V$, all of them hyperbolic, but $W_{\mu}^{u}(p) \cap W_{\mu}^{s}\left(q_{1}\right) \neq \Phi$ and transversal, 
(c) for $f_{\mu_{o}}, \mu_{o} \in U_{3}=\left\{\left(\mu_{1}, \mu_{2}\right) ; \mu_{1}>0\right\} p$ is the only fixed point in $V$, which is hyperbolic.

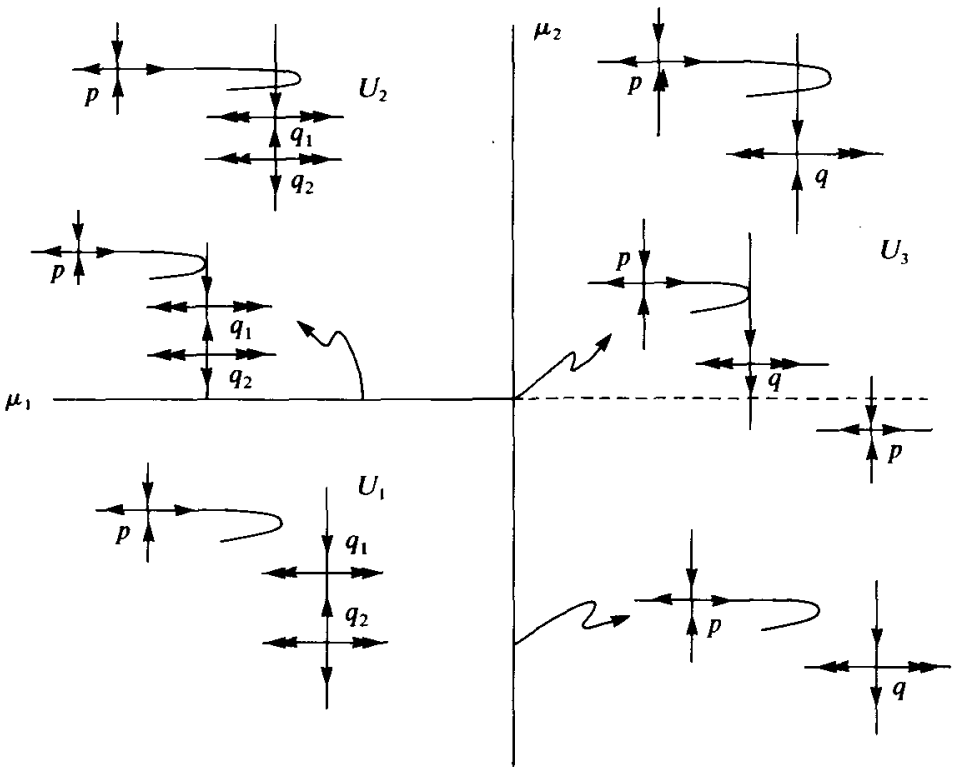

When $\mu$ lies in the semiaxis $\left\{\mu_{1}<0, \mu_{2}=0\right\}, W_{\mu}^{u}(p)$ meets $W_{\mu}^{s}\left(q_{1}\right)$ quasi-transversally along a unique orbit of tangency. This gives rise to a modulus of equivalence [8], namely $\log \beta / \log \alpha$.

Finally, the saddle-nodes appear only along the axis $\left\{\mu_{1}=0\right\}$, where we distinguish three classes of equivalence:

(a) for $f_{\left(0, \mu_{2}\right)}, \mu_{2}<0, W_{\mu}^{u}(p) \cap W_{\mu}^{c}(q)=\Phi$

(b) for $f_{\left(0, \mu_{2}\right)}, \mu_{2}>0, W_{\mu}^{u}(p)$ intersects $W^{s}(q)$ transversally

(c) at $f_{(0,0)}$ we have a tangency between $W_{0}^{u}(p)$ and $W_{0}^{s}(q)$.

\section{Mild equivalence}

In this section we will prove Theorems $A$ and $B$ from the Introduction.

As Theorem B is an easy consequence of Theorem A, we first show it follows from Theorem $A$.

\section{Proof of Theorem $B$}

(i) $f_{\mu} \in \mathscr{A}_{1}, \quad \mu \in U \subset \mathbf{R}^{2}$.

If $\tilde{f}_{\mu}$ is a nearby family in $\mathscr{A}_{1}$ and the central bifurcations $f_{(0,0)}$ and $\tilde{f}_{(0,0)}$ are equivalent, we claim that there exists a reparametrization $\rho: U \rightarrow \tilde{U}$ for which $\rho\left(U_{i}\right)=\tilde{U}_{i}, 1 \leq i \leq 3 ; \rho(0,0)=(0,0), \rho\left(0, \mu_{2}\right)=\left(0, \tilde{\mu}_{2}\right)$ and $\rho\left(\mu_{1}, \xi\left(\mu_{1}\right)\right)$ satisfies

$$
\frac{\log \beta}{\log \alpha}\left(\mu_{1}, \xi\left(\mu_{1}\right)\right)=\frac{\log \tilde{\beta}}{\log \tilde{\alpha}} \rho\left(\mu_{1}, \xi\left(\mu_{1}\right)\right) \text {. }
$$

Indeed, since

$$
\frac{\partial \beta}{\partial \mu_{2}}(0,0) \neq 0\left(\text { resp. } \frac{\partial \tilde{\beta}}{\partial \tilde{\mu}_{2}}(0,0) \neq 0\right) \text { then } \frac{\log \beta}{\log \alpha}\left(\operatorname{resp} \cdot \frac{\log \tilde{\beta}}{\log \tilde{\alpha}}\right)
$$


is monotonous along the curve of tangency. Moreover, as $\beta\left(\mu_{1}, \xi\left(\mu_{1}\right)\right) \rightarrow 1$ (resp. $\left.\tilde{\beta}\left(\tilde{\mu}_{1}, \xi\left(\tilde{\mu}_{1}\right)\right) \rightarrow 1\right)$ monotonously as $\mu_{1} \rightarrow 0$ we have

$$
\frac{\log \beta}{\log \alpha} \rightarrow 0\left(\operatorname{resp} \cdot \frac{\log \tilde{\beta}}{\log \tilde{\alpha}} \rightarrow 0\right) \quad \text { as } \mu_{1} \rightarrow 0 \text {. }
$$

This concludes the proof for $f_{\mu} \in \mathscr{A}_{1}$.

(ii) $f_{\mu} \in \mathscr{A}_{2}, \quad \mu \in U \subset \mathbf{R}^{2}$.

Again the monotonicity of the invariants along the corresponding curves of tangency (actually semiaxis) allow us to define a reparametrization $\zeta: U \rightarrow \tilde{U}$ satisfying $\zeta(0,0)=(0,0), \zeta\left(0, \mu_{2}\right)=\left(0, \tilde{\mu}_{2}\right), \zeta\left(U_{i}\right)=\tilde{U}_{i}, 1 \leq i \leq 3$, and

$$
\frac{\log \beta}{\log \alpha}\left(\mu_{1}, 0\right)=\frac{\log \tilde{\beta}}{\log \tilde{\alpha}} \zeta\left(\mu_{1}, 0\right) \text { for } \mu_{1}<0 \text {. }
$$

This concludes the proof of Theorem $B$.

Following [11] we consider the strong stable foliation $\mathscr{F}_{\mu}^{s s}, \mu \in U$, varying continuously with $\mu ; \pi_{\mu}^{s s}$ denotes the projection of a neighbourhood of $q$ on a central manifold $W_{\mu}^{c}$ for $q$ along the leaves of $\mathscr{F}_{\mu}^{s s}$.

We denote by $\mathscr{F}_{\mu}^{u}(p)$ (resp. $\mathscr{F}_{\mu}^{s}(p)$ ) the trivial unstable (resp. stable) foliation given by the linearizing coordinates at $p ; \pi_{\mu}^{u}$ (resp. $\pi_{\mu}^{s}$ ) is the corresponding projection on $W_{\mu}^{u}(p)\left(W_{\mu}^{s}(p)\right.$ resp.). We also denote by $\alpha_{\mu}$ the contracting eigenvalue at $p$.

The conclusion of Theorem $\mathrm{A}$ for $f \in S N Q T 2$ is an easy consequence of the proof in [5] and [11]. So we only deal with $f \in S N Q T 1$.

The goal now is to show that mild equivalence implies $\alpha=\tilde{\alpha}$ as stated in Theorem A.

Let $\left.f\right|_{W^{c}(q)}(x)=\varphi(x)=x+x^{2}+o_{3}(x)$ and define $N(x, \varphi)=\min \left\{k \in \mathbf{N}, \varphi^{k}(x) \geq \delta\right\}$ where $\delta>0$ is small enough.

Consider a sequence $\left\{z_{m}\right\}$ such that

(a) $z_{m} \rightarrow 1 \in W^{s}$ (p)as $m \rightarrow \infty$, identifying the point with its second coordinate.

(b) $y_{m}=\alpha^{m} z_{m} \rightarrow y_{0}$ as $m \rightarrow \infty, y_{0}$ is the point of tangency at $N$ and $y_{m}$ belongs to a transversal segment to $W^{u}(p)$ at $y_{0}$.

Let $x_{m}=A_{m} y_{m}$, so that $A_{m} \rightarrow A>0$, where we identify $x_{m}$ with its projection on $W^{u}(q)$ along the leaves of $\mathscr{F}^{s s}$, and $A_{m}, m \in \mathbf{N}$, are non zero constants.

Call $N_{m}(\varphi)=N\left(x_{m}, \varphi\right)$. Let us prove the following

Proposition 1. $\lim _{m \rightarrow \infty} \log N_{m}(\varphi) / m=-\log \alpha$.

Proof. As pointed out in [11], up to some smooth coordinate system, the map $\varphi(x)$ is the time-one map of a vector field of the form $X(x)=x^{2}+o_{3}(x)$. Let $X$, be the flow of $X$. Then

$$
t_{m} \leq N_{m} \leq t_{m}+1,
$$

where $X_{t_{m}}\left(x_{m}\right)=\delta$ and $N_{m}=N_{m}(\varphi)$.

If $X_{1}(x)=\frac{1}{2} x^{2}, X_{2}(x)=2 x^{2}$ and $t_{i, m}$ is defined by $X_{i, t_{i, m}}\left(x_{m}\right)=\delta$, the following inequality holds:

$$
t_{2, m} \leq t_{m} \leq N_{m} \leq t_{m}+1 \leq t_{1, m}+1
$$


As $t_{i, m}=\int_{x_{m}}^{\delta} X_{i}(x) d x$ we get

So,

$$
\frac{1}{2}\left[-\frac{1}{\delta}+\frac{1}{x_{m}}\right] \leq N_{m} \leq 2\left[-\frac{1}{\delta}+\frac{1}{x_{m}}\right]+1 .
$$

$$
\frac{1}{2}\left[-\frac{\alpha_{m} A_{m}}{\delta}+\frac{1}{z_{m}}\right] \leq \alpha^{m} A_{m} N_{m} \leq 2\left[-\frac{\alpha^{m} A_{m}}{\delta}+\frac{1}{z_{m}}\right]+\alpha^{m} A_{m}
$$

Taking logarithms,

$$
\begin{aligned}
\log \left[\frac{1}{2}\left(-\frac{\alpha^{m} A_{m}}{\delta}+\frac{1}{z_{m}}\right)\right] & \leq m \log \alpha+\log A_{m}+\log N_{m} \\
& \leq \log \left[2\left(-\frac{\alpha^{m} A_{m}}{\delta}+\frac{1}{z_{m}}\right)+\alpha^{m} A_{m}\right]
\end{aligned}
$$

Since $\alpha^{m} \rightarrow 0, z_{m} \rightarrow 1$ and $A_{m} \rightarrow A \neq 0$ as $m \rightarrow \infty$ we obtain

$$
\lim _{m \rightarrow \infty} \frac{\log N_{m}}{m}=-\log \alpha \text {. }
$$

Remark. If $\lim _{m \rightarrow \infty} \inf A_{m}=0$, the same computations show

\section{Proof of Theorem $A$}

$$
\liminf _{m \rightarrow \infty} \frac{\log N_{m}}{m} \geq-\log \alpha .
$$

(I) Necessity.

Let $h$ be a conjugacy between $f$ and $\tilde{f} \in S N Q T 1$ and consider the sequences $\left\{z_{m}\right\}$, $\left\{y_{m}\right\},\left\{x_{m}\right\}$ as above and their images under $h,\left\{\tilde{z}_{m}\right\},\left\{\tilde{y}_{m}\right\},\left\{\tilde{x}_{m}\right\}$. For simplicity assume $h(\delta)=\tilde{\delta} \in W_{c}(\tilde{q}), \tilde{\delta}$ small enough.

We will see that $\log \alpha \leq \log \tilde{\alpha}$ and then, considering $h^{-1}$, we get the reverse inequality.

We know that $x_{m}=A_{m} \alpha^{m}, A_{m} \rightarrow A>0$ as $m \rightarrow \infty$ and $\tilde{x}_{m}=\tilde{A}_{m} \tilde{\alpha}^{m}$, where $\tilde{A}_{m}$ is bounded from above. Observe that $\lim _{\inf _{m \rightarrow \infty}} \tilde{A}_{m}$ could be zero. In any case, as we remarked,

$$
\underset{m \rightarrow \infty}{\liminf } \frac{\log N_{m}(\tilde{\varphi})}{m} \geq-\log \tilde{\alpha}
$$

where $\tilde{\varphi}=\left.\tilde{f}\right|_{w_{(\tilde{q})}^{c}}$ and $N_{m}(\tilde{\varphi})=N\left(\tilde{x}_{m}, \tilde{\varphi}\right)$.

But, since $h$ is a conjugacy, $N\left(\tilde{x}_{m}, \tilde{\varphi}\right)=N\left(x_{m}, \varphi\right)$. Hence the last inequality together with Proposition 1 yield

$$
-\log \alpha \geq-\log \tilde{\alpha}
$$

(II) Sufficiency.

It will follow easily from the proof of Theorem C.

3. Strong equivalence for $f_{\mu} \in \mathscr{A}_{1}$

Several restrictions arise for strong equivalence between one parameter families [4]:

(1) $\left.f_{o}\right|_{w_{o}^{c}}$ is the time one map of a flow which has to be conjugate to the corresponding flow for $\left.\tilde{f}_{o}\right|_{w_{i}}$. In particular, the conjugacy $h_{o}$ is determined once it is defined at two points. It is differentiable at $W_{o}^{c} \backslash\{q\}$ and the lateral derivatives exist at $q$. 
(2) $h_{\mathrm{o}}$ must preserve $\mathscr{F}_{\mathrm{o}}^{s s}$, where $\mathscr{F}_{0}^{s s}$ is the strong stable foliation for $q$. Necessarily, these restrictions remain in the two parameter case. The proof of the next Lemma is in [5]:

COMPATIBILIZATION LEMMA. Let $p$ be a hyperbolic fixed point of saddle type for $f$ and $\Gamma$ be a $C^{1}$ curve transverse to $W^{u}(p)$ at $x_{o} \in D^{u}(p)$, where $D^{u}(p)$ is a fundamental domain for $W^{u}(p)$. Let $\tilde{f}$ be $C^{2}$ near $f$ and $\tilde{p}, \tilde{\Gamma}, \tilde{x}_{o}$ be similar objects for $\tilde{f}$. Let $h: \Gamma \rightarrow \tilde{\Gamma}$ be an homeomorphism, $h\left(x_{o}\right)=\tilde{x}_{o}, h(x) / x^{\delta} \rightarrow C$ as $x \rightarrow x_{o}, \delta=\log \tilde{\alpha} / \log \alpha, C$ a non zero constant. Take $\tilde{A}$ and $\tilde{B}$ tubular neighbourhoods of $\tilde{\Gamma}$ and $\tilde{f}^{-1}(\tilde{\Gamma})$ such that if $\partial \tilde{A}=\tilde{A}_{1} \cup \tilde{A}_{2}$ and $\partial \tilde{B}=\tilde{B}_{1} \cup \tilde{B}_{2}$ then $\tilde{f}^{-1}\left(\tilde{A}_{i}\right)=\tilde{B}_{i}, i=1,2$.

Then there is a continuous retraction $\pi: \tilde{N}^{s} \rightarrow W^{s}(\tilde{p})$, where $\tilde{N}^{s}$ is a neighbourhood of $W^{s}(\tilde{p})$ containing $D^{\mu}(\tilde{p}), \tilde{x}_{o} \in D^{u}(\tilde{p})$, satisfying the following properties:

(a) $\pi^{-1}(\tilde{p})=W^{u}(\tilde{p}) \cap \tilde{N}^{s}$

(b) $\pi$ is $\tilde{f}$ invariant, namely, $\pi(\tilde{f}(p))=\tilde{f}(\pi(p))$

(3) if $q \in \Gamma, \pi(h(q))=h\left(\pi^{s}(q)\right)$

(4) if $\tilde{U}$ is a domain of linearization for $\tilde{f}, \tilde{p} \in \tilde{U}$, then the fibres of $\pi$ on $U \cap \tilde{N}^{s}$ coincide with the fibres of $\tilde{\pi}^{s}$, except on the iterates of the strip bounded by $\tilde{A}_{1}$ and $\tilde{B}_{2}$

(5) in the interior of the strip bounded by $\tilde{A}_{1}$ and $\tilde{B}_{2}$ the fibres of $\pi$ are differentiable and transversal to the leaves of $\mathscr{F}^{s}(\tilde{p})$.

Moreover, $h$ extends to $W^{s}(p)$.

COROLlary 4. Suppose that $\mathscr{F}, \tilde{F}$ are fibrations defined on $N$, transversal to the fibrations $\pi$, $\tilde{\pi}$ except at $\Gamma, \tilde{\Gamma}$, where they have quadratic contact. Assume also that $\left.h\right|_{\Gamma \backslash\left\{x_{0}\right\}}$ is differentiable. Then $h$ extends to an homeomorphism in $N$ which is differentiable except along the fibres through $x_{0}$.

When no confusion is possible we still denote by $\mathscr{F}_{\mu}^{\mu}(\tilde{p})$ the foliation given by the Compatibilization Lemma and we say that $\mathscr{F}_{\mu}^{\mu}(\tilde{p})$ is obtained by compatibility with $h_{\mu}$.

Proof of Theorem $C$ for $f_{\mu} \in \mathscr{A}_{1}$ :

The plan is the following: we define a convenient reparametrization $\rho$ and take $\left.h_{\mu}\right|_{w_{\mu}^{c}}$ as in [11]. Choose $\mathscr{F}_{\mu}^{s s}(q)$ such that for $\mu=0$ it coincides with the foliation $\mathscr{F}_{o}^{s s}(q)$ already defined and let $\mathscr{F}_{\mu}^{u}(p)$ be the trivial fibration. We use the Compatibilization Lemma to obtain analogous suitable foliations for $\tilde{q}$ and $\tilde{p}$. These foliations intersect in $N$ along a unique curve of tangencies $\Gamma$. The homeomorphism $\left.h_{\mu}\right|_{w_{\mu}^{c}}$ induces a homeomorphism along $\Gamma$, which will be extended to $N$. Such an extension will vary continuously with $\mu$, will preserve $\mathscr{F}_{\mu}^{s s}$ at $\mu=\left(0, \mu_{2}\right)$, but not necessarily at other values of $\mu$.

We define $\rho \in C^{1}$ by

$$
\left\{\begin{array}{l}
\alpha_{\mu}-\tilde{\alpha}_{\mu}=0 \\
\beta_{\mu}-\tilde{\beta}_{\mu}=0 .
\end{array}\right.
$$

By the Implicit Function Theorem, and the fact that $f_{\mu}, \tilde{f}_{\mu} \in \mathscr{A}_{1}$, we can express $\tilde{\mu}_{i}=\tilde{\mu}_{i}\left(\mu_{1}, \mu_{2}\right)$ and inversely $\mu_{i}=\mu_{i}\left(\tilde{\mu}_{1}, \tilde{\mu}_{2}\right), i=1,2$. 
From the hypothesis it follows that $\rho$ maps the curve $\left(\mu_{1}, \xi\left(\mu_{1}\right)\right)$ onto $\left(\tilde{\mu}_{1}, \tilde{\xi}\left(\mu_{1}\right)\right)$ and maps $\left\{\mu_{1}=0\right\}$ onto $\left\{\tilde{\mu}_{1}=0\right\}$, i.e., $\rho$ preserves diffeomorphisms corresponding to tangencies and also diffeomorphisms exhibiting saddle node fixed points.

Let $\eta$ be the curve in $U$ given by

$$
\left.\eta=\left\{\left(\mu_{1}, \eta\left(\mu_{1}\right)\right) ; W_{\mu}^{u}(p) \text { meets } W_{\mu}^{s s}\left(q_{2}\right) \text { quasi-transversally }\right)\right\} .
$$

Note that $\eta\left(\mu_{1}\right) \cong-A \sqrt{\left|\mu_{1}\right|}$ (see $\left.\S 1\right)$.

We also note that $\rho$ does not necessarily map $\eta$ onto $\tilde{\eta}$.

Let $C=\left\{\left(\mu_{1}, \mu_{2}\right) ;-A_{2} \sqrt{\left|\mu_{1}\right|}<\mu_{2}<-A_{1} \sqrt{\left|\mu_{1}\right|}\right\}$ be such that $\eta, \rho^{-1}(\tilde{\eta})$ are contained in the interior of $C$, a wedge shaped neighbourhood of $\eta$ and $\rho^{-1}(\tilde{\eta})$.

When no confusion arises we will refer to the separatrix of $W_{\mu}^{u}(p) \backslash\{p\}$ that for some $\mu$ intersects the correspondent separatrix of $W_{\mu}^{s s}\left(q_{2}\right) \backslash\left\{q_{2}\right\}$ as $W_{\mu}^{u}(p)$ and $W_{\mu}^{s s}\left(q_{2}\right)$.

Let $\mathscr{F}_{\mu}^{s s}$ be a strong stable foliation such that:

(a) for each $\mu$, the space of leaves of $\mathscr{F}_{\mu}^{s s}$ is $W_{\mu}^{c}$

(b) $\mathscr{F}_{\mu}^{s s}$ extends the unique strong stable foliation defined on $W_{\mu}^{s}(q)$ for $\mu=\left(0, \mu_{2}\right)$

(c) $W_{\mu}^{s s}\left(q_{1}\right)$ and $W_{\mu}^{s s}\left(q_{2}\right)$ are leaves of $\mathscr{F}_{\mu}^{s s}$

(d) $\mathscr{F}_{\mu}^{s s}$ is invariant by $f_{\mu}$ and varies continuously with $\mu$

(e) for a fixed $\mu_{0}, F_{\mu_{0}}^{s s}(x) \in \mathscr{F}_{\mu_{o}}^{s s}$ is a $C^{\infty}$ disk varying continuously with $x$. Moreover, the tangent space to $F_{\mu_{0}}^{s s}(x)$ also varies continuously with $x$.

$\pi_{\mu}^{s s}$ denotes the projection of a neighbourhood of $\left\{q_{1}, q_{2}\right\}$ on $W_{\mu}^{c}$ along the leaves of $\mathscr{F}_{\mu}^{s s}$.

Let now $B_{\mu} \subset \Gamma$ be a segment transversal to $W_{\mu}^{s s}\left(q_{2}\right)$ and $K_{\mu}$ a fundamental neighbourhood for $W_{\mu}^{s s}\left(q_{2}\right)$ such that

(i) $\partial K_{\mu} \supset B_{\mu} \cup f_{\mu}\left(B_{\mu}\right)$

(ii) for $\mu \in \rho^{-1}(\tilde{\eta}), B_{\mu} \cap W_{\mu}^{s s}\left(q_{2}\right) \neq \Phi$ and $B_{\mu} \cap W_{\mu}^{u}(p) \neq \Phi$

(iii) $B_{\mu}=\Phi$ for $\mu \notin C$.

Take $C_{\mu}\left(q_{2}\right)=\bigcup_{n \in N} f_{\mu}^{n}\left(K_{\mu}\right)$. It is possible to choose $K_{\mu}$ for which $\partial C_{\mu}\left(q_{2}\right) \backslash B_{\mu}$ is an invariant curve. Furthermore, $C_{\mu}\left(q_{2}\right)$ depends continuously on $\mu$ and $C_{\mu}=\Phi$ when $\mu \notin C$.

It is easy to see that for each $\mu$, the intersection of $\partial C_{\mu}\left(q_{2}\right)$ with a leaf of $\mathscr{F}_{\mu}^{s s}$ is either a unique point or empty.

Let us define $h_{\mu}$.

For $\mu \notin C$ we may define $h_{\mu}$ at $W_{\mu}^{c}$ as a diffeomorphism satisfying:

$$
\begin{aligned}
& h_{\mu} \rightarrow h_{o} \text { as } \mu \rightarrow 0, \\
& h_{\mu}^{\prime}(x) \rightarrow h_{o}^{\prime}(x) \text { as } \mu \rightarrow 0, x \neq q
\end{aligned}
$$

and the lateral derivatives converge at $x=q$.

For $\mu \in C$ the definition is not as simple. Suppose $W_{\mu}^{u}(p) \cap \partial C_{\mu}\left(q_{2}\right) \cap N \neq \Phi$. This intersection contains at most two points $a_{\mu}$ and $b_{\mu}$. It is possible to define a $C^{1}$ homeomorphism $h_{\mu}$ at $W_{\mu}^{c}$ in such way that the normal derivatives of the homeomorphism induced by $h_{\mu} / w_{\mu}$ at $a_{\mu}$ and $b_{\mu}$ are the same, and different from zero. If the above intersection is empty we define $h_{\mu}$ at $W_{\mu}^{c}$ as a $C^{1}$ homeomorphism such that $h_{\mu} \rightarrow h_{o}$ as $\mu \rightarrow 0$. 
Remark that we may suppose that $h_{\mu}$, defined as above varies continuously with $\mu$.

Using $\mathscr{F}_{\mu}^{s s}$ we extend $\left.h_{\mu}\right|_{w_{\mu}^{c}}$ to $\Gamma \backslash C_{\mu}\left(q_{2}\right)$ and to $\partial C_{\mu}\left(q_{2}\right)$. We still denote this extension by $h_{\mu}$. We can choose $h_{\mu}$ so that $h_{\mu}\left(\partial C_{\mu}\left(q_{2}\right)\right)=\partial C_{\mu}\left(\tilde{q}_{2}\right)$ and the normal derivatives of $h_{\mu}$ at $a_{\mu}, b_{\mu}, \Gamma \cap W_{\mu}^{u}(p)$ vary continuously with $\mu$.

Inside $C_{\mu}\left(q_{2}\right)$ we proceed as follows.

We first define $h_{\mu}\left(B_{\mu}\right)=\tilde{B}_{\mu}$ and

$$
h_{\mu}\left(W_{\mu}^{u}(p) \cap C_{\mu}\left(q_{2}\right) \cap N\right)=W_{\mu}^{u}(\tilde{p}) \cap C_{\mu}\left(\tilde{q}_{2}\right) \cap \tilde{N} .
$$

Then we extend it to $C_{\mu}\left(q_{2}\right) \cap N$ in such way that the normal derivatives along $W_{\mu}^{u}(p) \cap C_{\mu}\left(q_{2}\right) \cap N$ are all equal.

Consider the foliation $\mathscr{F}_{\mu}^{u}(\tilde{p})$ obtained by compatibility with $\left.h_{\mu}\right|_{\Gamma \cup \partial C_{\mu}\left(q_{2}\right)}$.

Extend $h_{\mu}$ to $N-C_{\mu}\left(q_{2}\right)$ preserving $F_{\mu}^{s s}$ and $\mathscr{F}_{\mu}^{u}$. The extension of $h_{\mu}$ to a neighbourhood of $p, q_{1}$ and $q_{2}$ is obtained using the equation $f_{\mu}^{n} h_{\mu}=h_{\mu} \tilde{f}_{\mu}^{n}$. Since all the normal derivatives of $h_{\mu}$ along $W_{\mu}^{u}(p) \cap C_{\mu}\left(q_{2}\right) \cap N$ are equal and the equality $\alpha(\mu)=\tilde{\alpha} \rho(\mu)$ holds, $h_{\mu}$ extends to $W_{\mu}^{s}(p)$.

The continuity of $h_{\mu}$ on $\mu$ follows from the continuity on $\mu$ of $\left.h_{\mu}\right|_{w_{\mu}^{c}}, C_{\mu}\left(q_{2}\right)$, $\mathscr{F}_{\mu}^{s s}, \mathscr{F}_{\mu}^{u}$ and the normal derivatives along $W_{\mu}^{u}(p)$.

\section{Strong equivalence for $f_{\mu} \in \mathscr{A}_{2}$}

In this section we prove Theorem $\mathrm{C}$ for $f_{\mu} \in \mathscr{A}_{2}$.

Recall that for $\mu_{1}=0$ both $f_{\mu}$ and $\tilde{f}_{\mu}$ are the time one map of a vector field along $W_{\mu}^{c}$ and the conjugacy between these diffeomorphisms has to be also a conjugacy between the corresponding vector fields. So, $\left.h_{\mu}\right|_{w_{\mu}^{c}}$ is very rigid: it is uniquely determined by its values at two points in $W_{\mu}^{c}(q) \backslash\{q\}$, and it is also differentiable. If $\left\{a_{\mu}, b_{\mu}\right\}=N \cap W_{\mu}^{c}(q) \cap W_{\mu}^{u}(p)$, the differentiability of $h_{\mu}$ at these points implies that its extension to $W_{\mu}^{s}(p)$ has to be linear, that is, $\alpha_{\mu}=\tilde{\alpha}_{\mu}$. Moreover, the normal derivatives of $h_{\mu}$ at these points must be equal, i.e., $k\left(a_{\mu}\right)=k\left(b_{\mu}\right)$ in order that such an extension exists.

The plan of the proof is as follows:

(a) we use the results in [11] to define $h_{\mu} / W_{\mu}^{c}$ and the reparametrization

(b) in order to extend $h_{\mu}$ we define some special fibrations

(c) the region $R_{\mu}$ bounded by $W_{\mu}^{u}(p)$ and $W_{\mu}^{c}\left(q_{1}\right)$, for $\mu=\left(\mu_{1}, \mu_{2}\right), \mu_{2}>0$, is carefully analysed so as to guarantee the extension of $h_{\mu}$ to neighbourhoods of $p$ and $q_{1}$ as well as the continuity of $h_{\mu}$ on $\mu$.

We start by choosing convenient coordinates around $p(\mu)$ and $q_{1}(\mu)$.

Since the corresponding Sternberg condition is trivially satisfied, according to Takens [13], we fix $C^{r}$ coordinates, $r \geq 2$, for $f_{\mu}$ such that $f_{\mu}$ near $q_{1}$ is given by

$$
f_{\mu}(x, y)=\left(\varphi\left(x, \mu_{1}, \mu_{2}\right), b\left(x, \mu_{1}, \mu_{2}\right) y\right) \text {, }
$$

where $(x, 0) \in W_{\mu}^{c}\left(q_{1}\right)$ and $(0, y) \in W_{\mu}^{u u}\left(q_{1}\right)$ for $\mu_{1} \leq 0, \quad(\partial \varphi / \partial x)\left(0,0, \mu_{2}\right)=1$, $b\left(0, \mu_{1}, \mu_{2}\right)=\beta\left(\mu_{1}, \mu_{2}\right)$ for $\mu_{1} \leq 0$.

Analogously, by imposing the Sternberg condition on $f_{(0,0)}$ at $p$ (which holds in an open and dense subset) we fix $C^{r}$ linearizing coordinates, $r \geq 2$, on a neighbour- 
hood $V_{o}$ of $p$. Call $\mathscr{F}_{\mu}^{u}$ the trivial unstable fibration given by these coordinates and let $\pi_{\mu}^{u}: V_{o} \rightarrow W_{\mu}^{s}(p)$ be the projection along the leaves.

It is basic for our proof to fix a suitable conjugacy along $W_{\mu}^{c}$. All further constructions are based upon $h_{\mu} / W_{\mu}^{c}$. This is the subject of our next Lemma.

LEMMA 5. For $f_{\mu}, \tilde{f}_{\mu}$ there are a reparametrization $\rho(\mu)=\tilde{\mu}$ and a homeomorphism $h_{\mu} / W_{\mu}^{c}: W_{\mu}^{c} \rightarrow W_{\tilde{\mu}}^{c}$ such that

(a) $h_{\mu}$ is a conjugacy between $f_{\mu} / W_{\mu}^{c}$ and $\tilde{f}_{\tilde{\mu}} / W_{\mu}^{c}$, depending continuously on $\mu$

(b) for $\mu_{2} \geq 0,\left.(d / d y) \pi_{\mu}^{u}\left(h_{\mu}(y)\right)\right|_{y=a_{\mu}}=\left.(d / d y) \pi_{\mu}^{u}\left(h_{\mu}(y)\right)\right|_{y=b_{\mu}}$, where $\left\{a_{\mu}, b_{\mu}\right\}=$ $W_{\mu}^{c}(q) \cap W_{\mu}^{\mu}(p) \cap N$.

Proof. Given $f_{\mu}$ and $\tilde{f}_{\mu}$ let $X_{\mu}$ and $\tilde{X}_{\mu}$ be corresponding families of adapted vector fields defined on $W_{\mu}^{c} \times \mathbf{R}^{2}$.

Following [11] we choose a box

$$
U=\left\{(x, \mu) ;|x| \leq d, 0 \leq\left|\mu_{1}\right|+\left|\mu_{2}\right| \leq \varepsilon\right\}
$$

where $X(x, \mu)$ and $(\partial / \partial \mu) X(x, \mu)$ are positive on $U \backslash\{(0,0)\}$. We assume $\left\{a_{\mu}, b_{\mu}\right\} \subset$ $U, a_{\mu}<0, b_{\mu}<0$ and both are contained in a fundamental domain $D_{\mu}$ for $W_{\mu}^{c}$.

For $\mu_{1}>0$ we define $T_{\mu}$ by $X_{T_{\mu}}(-d, \mu)=(d, \mu)$. Observe that $T_{\mu}$ goes to infinity monotonously as $\mu_{1}$ goes to zero.

For $\mu_{2} \geq 0$, let $t_{\mu}$ satisfy $X_{t_{\mu}}\left(a_{\mu}\right)=b_{\mu}$.

In a similar way we define $\tilde{a} \mu, \tilde{b}_{\mu}, \tilde{t}_{\mu}$ and $\tilde{T}_{\mu}$.

Choice of the reparametrization $\rho$

(a) For $\mu_{1}>0$ and $\mu_{2} \geq 0, \rho$ is implicitly given by

$$
\left\{\begin{array}{l}
T_{\mu}=\tilde{T}_{\mu} \\
\alpha_{\mu}=\tilde{\alpha}_{\tilde{\mu}} .
\end{array}\right.
$$

(b) For $\mu_{1} \leq 0, \mu_{2} \geq 0, \rho$ is implicitly given by

$$
\left\{\begin{array}{l}
t_{\mu}=\tilde{t}_{\bar{\mu}} \\
\alpha_{\mu}=\tilde{\alpha}_{\tilde{\mu}} .
\end{array}\right.
$$

(c) For $\mu_{1}<0, \mu_{2}<0$ we extend $\rho$ with the property that $\beta_{\mu}=\tilde{\beta}_{\tilde{\mu}}$, and the semiaxis $\mu_{2}<0, \mu_{1}=0$ is preserved.

(d) For $\mu_{1} \geq 0, \mu_{2} \leq 0$ we proceed as follows.

Along the semiaxis $\left(\mu_{1}, 0\right), \mu_{1} \geq 0$, the reparametrization is already given in (b); also at $\left(0, \mu_{2}\right), \mu_{2} \leq 0$, the reparametrization is given in (c). Under generic conditions, namely

$$
\frac{\partial}{\partial x} \beta_{(0,0)}\left(q_{1}\right) \neq 0 \text { and } \frac{\partial}{\partial x} \tilde{\beta}_{(0,0)}\left(\tilde{q}_{1}\right) \neq 0 \text {, }
$$

it is possible to choose a reparametrization $\rho$ and families of points $x_{\mu} \in W_{\mu}^{c}, \tilde{x}_{\tilde{\mu}} \in W_{\tilde{\mu}}^{c}$ varying continuously with $u, \tilde{\mu}$ in the fourth quadrant verifying:

(d.1) at the boundary of the fourth quadrant we have

$$
\left\{\begin{array}{l}
x_{\left(0, \mu_{2}\right)}=q_{1}, \quad \tilde{x}_{\left(0, \tilde{\mu}_{2}\right)}=\tilde{q}_{1} \\
b\left(x_{\mu}, \mu\right)=\tilde{b}\left(\tilde{x}_{\tilde{\mu}}, \tilde{\mu}\right) .
\end{array}\right.
$$


(d.2) in the interior of the fourth quadrant we have

$$
\left\{\begin{array}{l}
b\left(x_{\mu}, \mu\right)=\tilde{b}\left(\tilde{x}_{\tilde{\mu}}, \tilde{\mu}\right) \\
T_{\mu}=\tilde{T}_{\tilde{\mu}} .
\end{array}\right.
$$

Observe that $\rho$ preserves both axis.

We now define $h_{\mu} / W_{\mu}^{c}$. Let $g_{\mu}$ be a conjugacy between $X_{\mu}, \tilde{X}_{\mu}$ such that $g_{\mu}\left(a_{\mu}\right)=\tilde{a}_{\bar{\mu}}$. We will define $h_{\mu}=\tilde{u}_{\tilde{\mu}}^{-1} g_{\mu} u_{\mu}$, where $u_{\mu}$ (resp. $\tilde{u}_{\tilde{\mu}}$ ) is a conjugacy between $f_{\mu}$ and $X_{\mu}$ (resp. $\tilde{f}_{\mu}$ and $\tilde{X}_{\tilde{\mu}}$ ), with $u_{\mu}(x)=x$ for $\mu=\left(0, \mu_{2}\right)$ (resp. $\tilde{u}_{\tilde{\mu}}(x)=x$ for $\left.\tilde{\mu}=\left(0, \tilde{\mu}_{2}\right)\right)$.

(a) For $\mu_{1}>0$ we choose $u_{\mu}, \tilde{\mu}_{\tilde{\mu}}$ such that

$$
\begin{gathered}
h_{\mu}\left(b_{\mu}\right)=\tilde{\mu}_{\tilde{\mu}}^{-1} g_{\mu} u_{\mu}\left(b_{\mu}\right)=\tilde{b}_{\tilde{\mu}}, \\
\left.\frac{d}{d y} \pi_{\mu}^{u}\left(\tilde{u}_{\tilde{\mu}}^{-1} g_{\mu} u_{\mu}\right)(y)\right|_{y=a_{\mu}}=\left.\frac{d}{d y} \pi_{\mu}^{u}\left(\tilde{u}_{\tilde{\mu}}^{-1} g_{\mu} u_{\mu}\right)(y)\right|_{y=b_{\mu}}, \\
u_{\mu}(x)=x \text { for } \mu=\left(0, \mu_{2}\right) \quad \text { and } \\
\tilde{u}_{\tilde{\mu}}(x)=x \text { for } \tilde{\mu}=\left(0, \tilde{\mu}_{2}\right) .
\end{gathered}
$$

The moduli conditions grant that these equations hold simultaneously at $\mu_{1}=0$. For $\mu_{1}>0$ we extend $u_{\mu}$ and $\tilde{u}_{\tilde{\mu}}$ continuously with $\mu$ since we are free to choose $u_{\mu}$ in $D_{\mu}$ and $\tilde{u}$ in $\tilde{D}_{\tilde{\mu}}$.

Remark that at $\mu=\left(\mu_{1}, 0\right) . a_{\mu}=b_{\mu}$, so that first two equalities hold trivially. For $\mu=\left(\mu_{1}, \mu_{2}\right), \mu_{2}<0$, they hold too, since $W_{\mu}^{u}(p) \cap W_{\mu}^{c}\left(q_{1}\right)=\Phi$.

(b) For $\mu_{1} \leq 0, \mu_{2} \geq 0$, we choose $u_{\mu}, \tilde{u}_{\tilde{\mu}}$ satisfying the same set of equations and preserving the corresponding fixed points.

Observe that in this quadrant the equations $X_{t_{\mu}}\left(a_{\mu}\right)=b_{\mu}$ and $\tilde{X}_{i_{\mu}}\left(\tilde{a}_{\mu}\right)=\tilde{b}_{\tilde{\mu}}$ hold by the definition of $\rho$.

(c) For $\mu_{1} \leq 0, \mu_{2}<0$, the only restrictions on $u_{\mu}$ and $\tilde{u}_{\tilde{\mu}}$ are

$$
\begin{array}{ll}
u_{\mu}(x)=x & \text { for } \mu=\left(0, \mu_{2}\right), \\
\tilde{u}_{\tilde{\mu}}(x)=x & \text { for } \tilde{\mu}=\left(0, \tilde{\mu}_{2}\right)
\end{array}
$$

and the preservation of the corresponding fixed points.

This completes the definition of $h_{\mu} \mid w^{c}$.

Let $\mathscr{F}_{\mu}^{c}(q)$ be a $C^{2}$ central foliation depending continuously on $\mu$ and $\pi_{c}: V_{q} \rightarrow$ $W_{q}^{u u}$ be the projection along the leaves ( $q$ is either a saddle-node or the hyperbolic saddle $\left.q_{1}\right)$. Call $\Gamma_{\mu} \subset N$ the unique $C^{1}$ curve of tangencies between $\mathscr{F}_{\mu}^{c}(q)$ and $\mathscr{F}_{\mu}^{u}(p)[5,11]$.

Consider $N \cap \pi_{u}^{-1}(y) \cap W_{\mu}^{c}$, which is either empty or consists of one or two points. In the last case, we call them $\left(x_{1}, y\right)$ and $\left(x_{2}, y\right), x_{1}<x_{2}$. Their images under $\left.h_{\mu}\right|_{w_{\mu}^{c}}$ are $\left(\tilde{x}_{1}, \tilde{y}_{1}\right)$ and $\left(\tilde{x}_{2}, \tilde{y}_{2}\right)$ where $\tilde{y}_{1}$ is generally different from $\tilde{y}_{2}$ due to the rigidity of $h_{\mu}$. So the image of an horizontal segment of $\mathscr{F}_{\mu}^{\mu}(p)$ may not be horizontal; thus $\mathscr{F}_{\mu}^{u}(p) \cap N$ and $\mathscr{F}_{\mu}^{u}(\tilde{p}) \cap \tilde{N}$ cannot be simultaneously preserved. Nevertheless, the equality of the normal derivatives guarantees the

Proposition 6. There is an invariant foliation $\Phi_{\mu}^{u}$ defined in a neighbourhood $\hat{V}_{\mu}$ of $\tilde{p}$, so that the projection along the leaves $\hat{\pi}_{\mu}^{u}: \hat{V} \rightarrow W_{\mu}^{s}(\tilde{p})$ satisfies

(a) $\hat{\pi}_{\mu}^{u}\left(h_{\mu}(x, y)\right)=h_{\mu}\left(\pi_{\mu}^{u}(x, y)\right)$ for $(x, y) \in\left(W_{\mu}^{s}(p) \cup W_{\mu}^{s}\left(q_{1}\right)\right) \cap \hat{V}$ 
(b) the segment joining $\left(\tilde{x}_{1}, \tilde{y}_{1}\right)$ and $\left(\tilde{x}_{2}, \tilde{y}_{2}\right)$ is contained in $\left(\hat{\pi}_{\mu}^{u}\right)^{-1}(\tilde{y})$ for some $\tilde{y} \in W_{\mu}^{s}(\tilde{p})$, that is, in a single fibre of $\hat{\pi}^{u}$

(c) the restriction of $\Phi_{\mu}^{u}$ to $N$ is $C^{1}$

(d) there is a unique $C^{1}$ curve $\tilde{\Gamma}_{\mu}$ of tangencies between $\Phi_{\mu}^{u}$ and $\mathscr{F}_{\mu}^{c}(\tilde{p})$ in $N$

(e) $\Phi_{\mu}^{u}$ varies continuously with $\mu$

(f) $\hat{\pi}_{\mu}^{u}(y) \equiv \pi_{\mu}^{u}(y)$ for $y \geq \tilde{s}(\mu), \tilde{s}(\mu)=o\left(\mu_{2}\right)$, where $\tilde{s}(\mu) \in \tilde{\Gamma}_{\mu}$ and will be determined later.

Proof. It is straightforward and follows from the proof of the Compatibilization Lemma. We observe that the choice of $\Phi_{\mu}^{u}$ and the preservation of $\mathscr{F}_{\mu}^{\mu}, \Phi_{\mu}^{u}$ and $\mathscr{F}_{\mu}^{c}$, $\mathscr{F}_{\tilde{\mu}}^{c}$ induce a definition $\left.h_{\mu}\right|_{\Gamma_{\mu}}: \Gamma_{\mu} \rightarrow \tilde{\Gamma}_{\mu}$.

The following proposition is a consequence of a result in [3]. Nevertheless, we include its proof for the sake of completeness.

Proposition 7. Let $\Gamma_{\mu}, \tilde{\Gamma}_{\mu}$ be $C^{1}$ curves parametrized by $(-\varepsilon, \varepsilon) \subseteq \mathbf{R}, \varepsilon>0$, transversal to $W_{\mu}^{c}(q)$ at 0 and $g_{\mu}: \Gamma_{\mu} \rightarrow \tilde{\Gamma}_{\mu}$ a homeomorphism such that $g_{\mu}(y) / y^{\lambda} \rightarrow c(\mu)$ as $y \rightarrow 0$, $\lambda=(\log \tilde{\beta} / \log \beta)(\mu)$. Then $g_{\mu}$ extends to the closure of $\bigcup_{n \geq 0} f_{\mu}^{n}\left(\Gamma_{\mu}\right) \cup W_{\mu}^{u u}\left(q_{1}\right)$.

Proof. The diffeomorphisms $f_{\mu}, \tilde{f}_{\dot{\mu}}$ are partially linearized:

$$
\begin{aligned}
& f_{\mu}(x, y)=\left(\mu_{1}+x+a_{\mu} x^{2}, b_{\mu}(x) y\right) \\
& \tilde{f}_{\mu}(x, y)=\left(\tilde{\mu}_{1}+x+\tilde{a}_{\mu} x^{2}, \tilde{b}_{\mu}(x) y\right) .
\end{aligned}
$$

Call $\pi_{\mu}^{1}, \pi_{\mu}^{2}$ (resp. $\tilde{\pi}_{\mu}^{1}, \tilde{\pi}_{\mu}^{2}$ ) these coordinates.

We take $\mathscr{F}_{\mu}^{c}$ and $\mathscr{F}_{\tilde{\mu}}^{c}$ invariant foliations such that $W_{\mu}^{c}\left(q_{1}\right)$ and $W_{\tilde{\mu}}^{c}\left(\tilde{q}_{1}\right)$ are leaves. We also take $\mathscr{F}_{\mu}^{\mu u}$ and $\mathscr{F}_{\bar{\mu}}^{\mu u}$ strong invariant foliations such that $\Gamma_{\mu}$ and $\tilde{\Gamma}_{\mu}$ are leaves.

The only point of this proof is the convergence of some infinite products. To get this we define the following conjugacy $G_{\mu}$ :

(a) $h_{\mu}\left|w_{\mu}^{c}=h_{\mu}\right| w_{\mu}^{c}$.

(b) $\left.G_{\mu}\right|_{\pi_{1}^{-1}\left(\pi_{1}\left(q_{1}\right)\right)}\left(\pi_{1} q_{1}, y\right)=\left(\pi_{1} \tilde{q}_{1}, c_{\mu} y^{\lambda}\right)$. Observe that for $\mu_{1} \leq 0, \pi_{1}^{-1}\left(\pi_{1}\left(q_{1}\right)\right)=$ $W^{u u}\left(q_{1}\right)$

(c) it preserves $\mathscr{F}_{\mu}^{c}, \mathscr{F}_{\mu}^{c}$ and $\mathscr{F}_{\mu}^{\mu u}, \mathscr{F}_{\mu}^{\mu u}$.

Now consider a sequence $\left(x_{\mu}, y_{\mu}\right) \in \Gamma_{\mu}$ for which $f_{\mu}^{k_{\mu}}\left(x_{\mu}, y_{\mu}\right) \rightarrow\left(w_{o}, z_{o}\right)$ as $k_{\mu} \rightarrow+\infty$, $\left(w_{o}, z_{o}\right) \in W_{\mu}^{u}\left(q_{1}\right)$. Observe that $\left(w_{o}, z_{o}\right)$ does not necessarily belong to $W_{\mu}^{u u}\left(q_{1}\right)$. Then

$$
y_{\mu} \prod_{j=0}^{k_{\mu}-1} b_{\mu}\left(f_{\mu}^{j}\left(x_{\mu}, y_{\mu}\right)\right) \rightarrow z_{o}
$$

and

$$
c_{\mu} y_{\mu}^{\lambda} \prod_{j=0}^{k_{\mu}-\lambda} \tilde{b}_{\mu}\left(\tilde{f}_{\mu}^{j}\left(\tilde{x}_{\mu}, \tilde{y}_{\mu}\right)\right) \rightarrow \varphi_{\mu}\left(w_{o}, z_{o}\right),
$$

where $c_{\mu} \rightarrow c_{\mu_{0}}$ as $\mu \rightarrow \mu_{o}, \varphi_{\mu}\left(x_{o}, y_{o}\right)^{\prime}=\tilde{\pi}_{2}\left(G_{\mu}\left(w_{o}, z_{o}\right)\right) \equiv \tilde{z}_{o},\left(\tilde{x}_{\mu}, \tilde{y}_{\mu}\right)=G_{\mu}\left(x_{\mu}, y_{\mu}\right)$. In particular, $\varphi_{\mu}\left(w_{o}, z_{o}\right)=C_{\mu_{i}} z_{o}^{\lambda}$ if $\left(w_{o}, z_{o}\right) \in W_{\mu}^{u u}\left(q_{1}\right)$. So,

$$
\prod_{j=0}^{k_{\mu}-1} \frac{b_{\mu}^{\lambda}\left(f_{\mu}^{j}\left(x_{\mu}, y_{\mu}\right)\right)}{\tilde{b}_{\mu}\left(\tilde{f}^{j}\left(\tilde{x}_{\mu}, \tilde{y}_{\mu}\right)\right)} \rightarrow C_{\mu_{o}} \frac{z_{o}^{\lambda}}{\varphi_{\mu}\left(w_{o}, z_{o}\right)} .
$$

Set $\left.g_{\mu}\right|_{w_{c}^{\mu}}=\left.h_{\mu}\right|_{w_{\mu}}$. We claim that $g_{\mu}$ extends to the closure of $\bigcup_{n \geq 0} f_{\mu}^{n}\left(\Gamma_{\mu}\right) \cup$ $W_{\mu}^{u \mu}\left(q_{1}\right)$. Indeed, for $\mu_{0}=\left(\mu_{1}, \mu_{2}\right), \mu_{1}>0$ we have $\bigcup f_{\mu}^{n}\left(\Gamma_{\mu}\right)=\bigcup f_{\mu}^{n}\left(\Gamma_{\mu}\right)$ and $W_{\mu}^{u}\left(q_{1}\right)$ is not defined. So, trivially $g_{\mu}$ extends and it is continuous. 
Thus, the interesting case is when $\mu_{o}=\left(0, \mu_{2}\right)$. Take a sequence $\left(x_{\mu}, y_{\mu}\right) \in \Gamma_{\mu}$ as before, with

$$
f_{\mu}^{k_{\mu}}\left(x_{\mu}, y_{\mu}\right)=\left(w_{\mu}, z_{\mu}\right) \rightarrow\left(w_{o}, z_{o}\right)
$$

Note that

$$
\begin{aligned}
\tilde{\pi}_{2}\left(\tilde{f}_{\mu}^{k_{\mu}}\left(g_{\mu}\left(x_{\mu}, y_{\mu}\right)\right)\right. & =\prod_{j=0}^{k_{\mu}-1} \tilde{b}_{\mu}\left(\tilde{f}_{\mu}^{j}\left(g_{\mu}\left(x_{\mu}, y_{\mu}\right)\right)\right) \frac{g_{\mu}\left(x_{\mu}, y_{\mu}\right)}{y_{\mu}^{\lambda}} \cdot y_{\mu}^{\lambda} \\
& =\prod_{j=0}^{k_{\mu}-1} \frac{\tilde{b}_{\mu}\left(\tilde{f}_{\mu}^{j}\left(g_{\mu}\left(x_{\mu}, y_{\mu}\right)\right)\right)}{b_{\mu}^{\lambda}\left(f_{\mu}^{j}\left(x_{\mu}, y_{\mu}\right)\right)} \cdot z_{\mu}^{\lambda} \cdot \frac{g_{\mu}\left(x_{\mu}, y_{\mu}\right)}{y_{\mu}^{\lambda}} .
\end{aligned}
$$

As $z_{\mu}$ converges to $z_{o}, g_{\mu}\left(x_{\mu}, y_{\mu}\right) / y_{\mu}^{\lambda} \rightarrow C(\mu)$ by hypothesis and

$$
\prod_{j=0}^{k_{\mu}-1} \frac{\tilde{b}_{\mu}\left(\tilde{f}_{\mu}^{j}\left(g_{\mu}\left(x_{\mu}, y_{\mu}\right)\right)\right)}{b_{\mu}^{\lambda}\left(f_{\mu}^{j}\left(x_{\mu}, y_{\mu}\right)\right)}
$$

converges by $(6.1)$, our result holds.

Important remark. For $\mu=\left(0, \mu_{2}\right)$ we can continuously extend $g_{\mu}$ to $W_{\mu}^{u}\left(q_{1}\right)$, preserving $\mathscr{F}_{\mu}^{u \mu}\left(q_{1}\right)$ and $\mathscr{F}_{\mu}^{c}\left(q_{1}\right)$. The same proof grants the continuity of $g_{\mu}$ with $\mu$.

The following two propositions will give $h_{\mu}$ on the second and third quadrants. To do so we distinguish some regions where we define special foliations.

Proposition 8. For $\mu_{1} \leq 0$ and $\mu_{2}>0$ there exists an homeomorphism $h_{\mu}$ defined on $N$ with the following properties:

(a) $\left.h_{\mu}\right|_{\Gamma_{\mu}}$ coincides with the homeomorphism defined in Proposition 6,

(b) it has an extension to a neighbourhood of $p$ and to a neighbourhood of $q_{1}$ such that $\left.h_{\mu}\right|_{w_{\mu}^{s}(p)}$ is linear and $\left.h_{\mu}\right|_{w_{\mu}^{\prime \prime}}\left(q_{1}\right)$ is logarithmically linear.

Proof. We first construct three fibrations $\mathscr{F}_{1}, \mathscr{F}_{2}$ and $\mathscr{F}_{3}$ in $N$. Let $\left\{c_{\mu}\right\}=\Gamma_{\mu} \cap$ $W_{\mu}^{s}\left(q_{1}\right) \cap N$, identifying this point with its projection $\pi_{\mu}^{u}\left(c_{\mu}\right)$ and consider

$$
\begin{aligned}
& r=r(\mu)=c_{\mu}-\mu_{2}^{2}, \\
& s_{o}=s_{o}(\mu)=c_{\mu}=\frac{3}{2} \mu_{2}^{2}, \\
& s_{1}=s_{1}(\mu)=c_{\mu}-\frac{\mu_{2}^{2}}{2}, \\
& s_{2}=s_{2}(\mu)=c_{\mu}+u_{2}^{2} .
\end{aligned}
$$

Observe that $s_{o}<r<s_{1}<s_{2}$, so that the leaves $L_{1}$ and $L_{2}$ of $\mathscr{F}_{\mu}^{c}\left(q_{1}\right)$ passing through $s_{1}$ and $s_{2}$ are the boundary of a neighbourhood of $W_{\mu}^{s}\left(q_{1}\right)$. Call $R_{i}=R_{i}(\mu)$ the region bounded by $W_{\mu}^{u}(p)$ and $L_{i}, i=1,2$.

Let $F_{\mu}^{u}(r) \in \mathscr{F}_{\mu}^{u}(p)$ be the leaf at $r$ and call $r_{i}=r_{i}(\mu), i=1,2$, the elements of $F_{\mu}^{u}(r) \cap L_{1}$.

Define $F_{1}=F_{1}(\mu)$ as the union of the vertical segment joining $r_{1}$ to $L_{2}$ with the arc of $L_{1}$ connecting $r_{1}$ to $W_{\mu}^{u}(p)$. Analogously we define $F_{2}$.

Let $R_{3}=R_{3}(\mu)$ be the region in $N$ bounded by $F_{1}, F_{2}, L_{2}$, that contains $\Gamma_{\mu} \cap$ $W_{\mu}^{c}\left(q_{1}\right)$. 


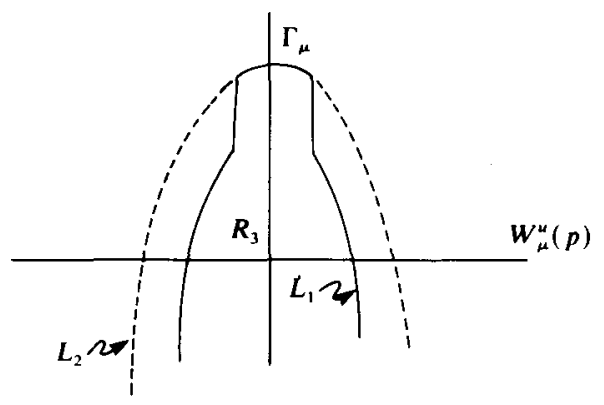

It is possible to define a continuous fibration $\mathscr{F}_{3}=\mathscr{F}_{3}(\mu)$ in $R_{3}$ with the following properties:

(a) $\Gamma \cap R_{3}, F_{1}$ and $F_{3}$ are fibres,

(b) $\mathscr{F}_{3}$ is topologically transversal to $\mathscr{F}_{\mu}^{c}\left(q_{1}\right)$ and $\mathscr{F}_{\mu}^{u}(p)$ in the interior of $R_{3} \cap$ $\left\{\pi_{\mu}^{\mu}(y) \geq 0\right\}$

(c) in $R_{3} \cap\left\{\pi_{\mu}^{u}(y) \leq 0\right\}$ it coincides with $\mathscr{F}_{\mu}^{c}\left(q_{1}\right)$, so that $\Gamma$ is a singular fibre.

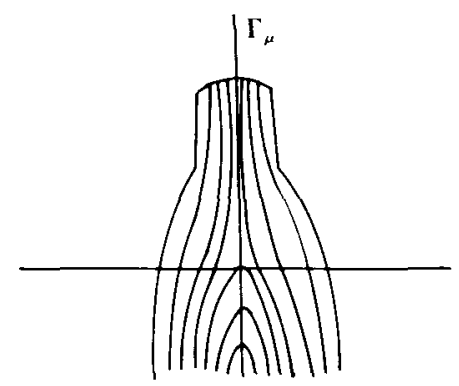

Let $R_{4}=R_{4}(\mu)=\left\{y \in N ; 0 \leq \pi_{u}(y) \leq \pi_{u}\left(s_{o}\right)\right\}$ and define $\mathscr{F}_{1}=\mathscr{F}_{1}(\mu)$ as:

(a) the restriction of $\mathscr{F}_{1}$ to $R_{1} \cup R_{4} \cup\left(N \backslash R_{2}\right)$ coincides with $\mathscr{F}_{\mu}^{\mu}(p)$

(b) the restriction of $\mathscr{F}_{1}$ to $R_{2} \backslash\left(R_{4} \cup R_{3}\right)$ is a $C^{1}$ foliation transversal to $\mathscr{F}_{\mu}^{c}\left(q_{1}\right)$ such that $F_{1}, F_{2}$ and the two segments $\left(\pi_{\mu}^{u}\right)^{-1}\left(\pi_{\mu}^{u}\left(s_{o}\right)\right) \cap R_{2} \backslash R_{1}$ are leaves.

Remark that $\mathscr{F}_{1}$ is not defined in $R_{3} \backslash R_{1}$.

Finally, take $\mathscr{F}_{2}=\mathscr{F}_{2}(\mu)$ as $\mathscr{F}_{\mu}^{c}\left(q_{1}\right)$ in $\left(N \backslash R_{1}\right) \cup\left(R_{1} \backslash R_{4}\right)$ and it is not defined in $R_{1} \cap R_{4}$.

In order to construct analogous objects for $\tilde{f}_{\mu}$ we need a definition of $\left.h_{\mu}\right|_{\Gamma}$, which now follows.

We set $h_{\mu}(y)=A(\mu) y^{\lambda}$ for $y \in W^{u u}\left(q_{1}\right)$. This induces a definition of $\left.h_{\mu}\right|_{\Gamma \backslash R_{1}}$, via projection along $\mathscr{F}_{\mu}^{c}\left(q_{1}\right), \tilde{\mathscr{F}}_{\mu}^{c}\left(\tilde{q}_{1}\right)$.

Recall that $\left.h_{\mu}\right|_{\Gamma \cap R_{1}}$ is given by the preservation of $\mathscr{F}_{\mu}^{\mu}, \Phi_{\mu}^{u}$ and $\mathscr{F}_{\mu}^{c}, \tilde{\mathscr{F}}_{\mu}^{c}$ together with $\left.h_{\mu}\right|_{w_{\mu}}$. We restrict this definition for $y \in \Gamma \cap R_{1}, y \leq r$, and complete it along $\Gamma \cap R_{1}$, for $\mu_{2}>0$, by continuity on $y$ and $\mu$.

The regions $\tilde{R}_{i}, 1 \leq i \leq 4$, and the fibrations $\tilde{\mathscr{F}}_{i}, 1 \leq i \leq 3$, are constructed analogously to $R_{i}$ and $\mathscr{F}_{i}$, respectively, except for the fact that $\Phi_{\mu}^{u}$ takes the place of $\mathscr{F}_{\mu}^{u}(\tilde{p})$, where $\Phi_{\mu}^{u}$ is given by Proposition 6 and $\tilde{r}=\tilde{r}(\mu)=h_{\mu}\left(r_{\mu}\right)$, $\tilde{s}_{i}=\tilde{s}_{i}(\mu)=h_{\mu}\left(s_{i}\right), 0 \leq i \leq 2$. 
Let us define $h_{\mu}$ on $N$.

Step 1 . Since the family $\left.h_{\mu}\right|_{\Gamma}$ is already defined and $h_{\mu}(y)=A(\mu) y^{\lambda}$ for $y \in W_{\mu}^{u u}\left(q_{1}\right)$, it induces a definition on $N \cap W^{u}(p)$ and $\left(\pi_{\mu}^{u}\right)^{-1}\left(\pi_{\mu}^{u}(r)\right) \cap R_{1}$ via $\mathscr{F}_{3}, \tilde{\mathscr{F}}_{3}$.

Note that $\left.h_{\mu}\right|_{W_{\mu}^{c}\left(q_{1}\right)}$ gives another correspondence between $\mathscr{F}_{3}$ and $\tilde{F}_{3}$, which induces a definition of $h_{\mu}$ on $L_{1} \cap\left\{\pi_{\mu}^{u}(y) \geq \pi_{\mu}^{u}\left(s_{o}\right)\right\}$.

We extend $h_{\mu}$ to $R_{1} \backslash R_{4}$ preserving the definition already given along $\Gamma$, continuously depending on $\mu$. Observe that $h_{\mu}$ may not preserve $\mathscr{F}_{3}$ in $R_{1} \backslash R_{4}$.

Step 2. Extend $h_{\mu}$ to $N$ preserving the above fibrations $\mathscr{F}_{1}, \tilde{\mathscr{F}}_{i}, 1 \leq i \leq 3$, by their identification through $\left.h_{\mu}\right|_{\Gamma \cup w_{\mu}^{s}\left(q_{1}\right)}$.

Step 3. Extend to $\bigcup_{n \in Z} f_{\mu}^{n}(N)$ by the equation

$$
h_{\mu} f_{\mu}^{n}=\tilde{f}_{\mu}^{n} h_{\mu} .
$$

Along $W_{\mu}^{s}(p)$ the conjugacy is given by $h_{\mu}(x)=k(\mu) x$, in the linearizing coordinates. This extension is continuous due to the definition of $h_{\mu}$ near $W_{\mu}^{u}(p)$ and the preservation of $\mathscr{F}_{1}, \tilde{\mathscr{F}}_{1}$.

Finally we extend $h_{\mu}$ to a neighbourhood of $W^{u \mu}\left(q_{1}\right)$ by preserving $\mathscr{F}_{\mu}^{u \mu}, \tilde{\mathscr{F}}_{\mu}^{u u}$ and $\mathscr{F}_{\mu}^{c}, \tilde{\mathscr{F}}_{\mu}^{c}$.

Proposition 9. For $\mu_{1} \leq 0$ and $\mu_{2} \leq 0$ there exists an homeomorphism $h_{\mu}$ defined on $N$ with the following properties:

(a) $\left.h_{\mu}\right|_{\Gamma}$ coincides with the homeomorphism defined on Proposition 6,

(b) it has an extension to a neighbourhood of $p$ and to a neighbourhood of $q_{1}$ such that $\left.h_{\mu}\right|_{w_{\mu}^{s}(p)}$ is logarithmically linear and $\left.h_{\mu}\right|_{w_{\mu}^{\prime \prime \prime}\left(q_{1}\right)}$ is linear.

Proof. Recall that for $\mu_{1} \leq 0, \mu_{2} \leq 0$ the reparametrization verifies $\beta_{\mu}=\tilde{\beta}_{\tilde{\mu}}$.

Define $\left.h_{\mu}\right|_{\Gamma}(y)$, for $y \leq c_{\mu}$, through the homeomorphism induced by $\left.h_{\mu}\right|_{w_{\mu}^{c}}$ and the preservation of $\mathscr{F}_{\mu}^{u}, \Phi_{\mu}^{u}$. For $y \geq s_{2}(\mu), h_{\mu}(y)=A_{\mu} y^{\log \dot{\alpha} / \log \alpha}$, and extend it diffeomorphically to all of $\Gamma$.

Since $\left.h_{\mu}\right|_{\Gamma}$ is differentiable at $\Gamma \cap W^{c}\left(q_{1}\right)$ and $\beta_{\mu}=\tilde{\beta}_{\tilde{\mu}}$, applying Proposition 7 we obtain a conjugacy on $\bigcup_{n \geq 0} f_{\mu}^{n}(\Gamma) \cup W_{\mu}^{u \mu}\left(q_{1}\right)$ which extends $\left.h_{\mu}\right|_{\Gamma}$. This conjugacy turns out to be linear along $W_{\mu}^{u u}\left(q_{1}\right)$.

Modify $\tilde{\mathscr{F}}_{\mu}^{c}$ by compatibility with $\left.h_{\mu}\right|_{\Gamma}$ and extend $\left.h_{\mu}\right|_{W_{\mu}^{u t}\left(q_{1}\right)}$ to $W_{\mu}^{u}\left(q_{1}\right)$ by preserving $\mathscr{F}_{\mu}^{c}, \tilde{\mathscr{F}}_{\mu}^{c}$ and $\mathscr{F}_{\mu}^{u}, \Phi_{\mu}^{u}$.

The extension of $h_{\mu}$ to a neighbourhood of $W_{\mu}^{s}(p)$ is a consequence of the definition of $\left.h_{\mu}\right|_{\Gamma}$ and the preservation of $\mathscr{F}_{\mu}^{u}$ and $\Phi_{\mu}^{u}$.

Proof of Theorem $C$ for $f_{\mu} \in \mathscr{A}_{2}$. In Propositions 8 and 9 we defined the homeomorphism $h_{\mu}$ for $\mu_{1} \leq 0$. Here we extend it to $\mu_{1}>0$ and prove continuity, using the same notations as before.

Recall that $\left.h_{\mu}\right|_{w_{\mu}^{c}}$ is already defined. In the first place, consider $\mu$ in the first quadrant. Take $\mathscr{F}_{\mu}^{\mu u}$ a strong unstable foliation for $f_{\mu}$ at $q_{1}$, continuous with $\mu$, transversal to $\mathscr{F}_{\mu}^{u}(p)$.

Let $S_{\mu_{2}}=R_{2} \backslash R_{1}$ and $S_{\mu_{1}}$ be strips around $W_{\mu}^{c}\left(q_{1}\right) \cap N$, where $S_{\mu_{1}}$ is chosen with the property that the closure of its saturation by $f_{\mu}$ contains a fixed segment of $F_{\mu}^{u \mu}\left(q_{1}\right)\left(F_{\mu}^{u u}\left(q_{1}\right)\right.$ is the fibre of $\mathscr{F}_{\mu}^{\mu u}$ at $\left.q_{1}\right)$. Observe that the width of $S_{\mu_{1}}$ goes to zero with $\mu_{1}$. Consider analogous objects for $\tilde{f}_{\mu}$. 
Define a fibration $\mathscr{F}_{4}=\mathscr{F}_{4}(\mu)$ in $S_{\mu_{2}}$ such that:

(a) it is transversal to $W_{\mu}^{c}\left(q_{1}\right)$

(b) it coincides with $\mathscr{F}_{\mu}^{u}$ in $\left\{\pi_{u}(y) \leq \pi_{u}\left(s_{o}\right)\right\}$

(c) it coincides with $\mathscr{F}_{3}$ in $R_{3} \cap S_{\mu_{2}}$.
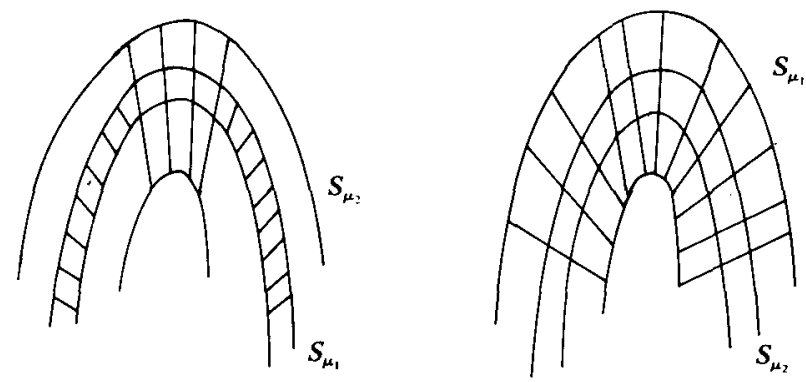

In a similar way we construct $\tilde{F}_{4}$, replacing $\mathscr{F}_{\mu}^{u}$ by $\Phi_{\mu}^{u}$.

Note that the definition of $\left.h_{\mu}\right|_{w_{\mu}^{c}}$ and the correspondence between $\mathscr{F}_{\mu}^{u}$ and $\Phi_{\mu}^{u}$, $\mathscr{F}_{\mu}^{\mu u}$ and $\tilde{F}_{\mu}^{u \mu}$ fix $h_{\mu}$ in a neighbourhood of $W_{\mu}^{c}$. Since we want $h_{\mu}$ to enjoy some additional properties, inside $\tilde{S}_{\mu_{1}}$, we choose a special strong unstable foliation $\tilde{\mathscr{F}}_{\mu}^{\mu}$ :

(a) it is transversal to $\Phi_{\mu}^{u}$

(b) it is the restriction of $\tilde{\mathscr{F}}_{3}$ to $\tilde{S}_{\mu_{1}}$, when $\tilde{S}_{\mu_{1}} \subseteq \tilde{S}_{\mu_{2}}$

(c) it is an extension of $\tilde{\mathscr{F}}_{3}$ when $\tilde{S}_{\mu_{1}} \supseteq \tilde{S}_{\mu_{2}}$

(d) for $w \in W_{\mu}^{c}\left(q_{1}\right), \pi_{u}(w) \leq \pi_{u}\left(s_{o}\right)$, we select $\tilde{F}^{u u}\left(h_{\mu}(w)\right)$ for which the homeomorphism

$$
h_{\mu, w}: F_{\mu}^{u u}(w) \rightarrow \tilde{F}_{\mu}^{u u}\left(h_{\mu}(w)\right)
$$

induced via projection of $\mathscr{F}_{\mu}^{u}$ on $\mathscr{F}_{\mu}^{u u}$ satisfies $h_{\mu, w}(y) / y^{\lambda} \rightarrow h(\mu)$ as $y \rightarrow 0$, where $k(\mu)$ depends continuously on $\mu, k(\mu) \neq 0$ for every $\mu$.

We are now ready to define $h_{\mu}$ in $N$. Along $\Gamma$ we follow the definition already given in Propositions 8 and 9.

For $\mu_{2} \geq 0$ we proceed as follows:

(a) in $R_{1} \cap N \backslash S_{\mu_{1}}$ we preserve $\mathscr{F}_{3}, \tilde{\mathscr{F}}_{3}$ and $\mathscr{F}_{\mu}, \Phi_{\mu}^{\mu}$

(b) in $N \backslash R_{2} \backslash S_{\mu_{2}}$ we preserve $\mathscr{F}_{\mu}^{c}, \tilde{F}_{\mu}^{c}$ and $\mathscr{F}_{\mu}^{u}, \Phi_{\mu}^{u}$

(E) if $S_{\mu_{1}} \subseteq S_{\mu_{2}}$ we preserve $\mathscr{F}_{\mu}^{c}, \tilde{\mathscr{F}}_{\mu}^{c}$ and $\mathscr{F}_{4}, \tilde{\mathscr{F}}_{4}$,

(d) if $S_{\mu_{1}} \supseteq S_{\mu_{2}}$ we preserve $\mathscr{F}_{\mu}^{c}, \tilde{\mathscr{F}}_{\mu}^{c}$ and $\mathscr{F}_{4}, \tilde{\mathscr{F}}_{4}$ inside $S_{\mu_{2}}$.

We preserve $\mathscr{F}_{\mu}^{u}, \Phi_{\mu}^{u}$ and $\mathscr{F}_{\mu}^{u u}, \tilde{\mathscr{F}}_{\mu}^{u u}$ outside $S_{\mu_{2}}$, but now the correspondence between the pieces of the fibres of the last pair of foliations is given by the homeomorphism defined on the boundary $\partial S_{\mu_{2}}=L_{1} \cup L_{2}$.

For $\mu_{2}<0, \mu_{1} \geq 0$, we recall that $\left.h_{\mu}\right|_{\Gamma}$ is differentiable except at $\Gamma \cap W_{\mu}^{u}(p)$.

We modify $\tilde{\mathscr{F}}_{\mu}^{c}$ by compatibility with $\left.h_{\mu}\right|_{\mathrm{r}}$ and extend $\left.h_{\mu}\right|_{\Gamma}$ to $N$ by preserving $\mathscr{F}_{\mu}^{u}, \Phi_{\mu}^{u}$ and $\mathscr{F}_{\mu}^{c}, \tilde{\mathscr{F}}_{\mu}^{c}$.

In $N$, this homeomorphism is differentiable except at the leaves through $\Gamma \cap$ $W_{\mu}^{u}(p)$. By Corollary 4 , a foliation $\mathscr{F}_{\mu}^{u u}$ in $N$, transversal to $\mathscr{F}_{\mu}^{c}$ and extending $\Gamma$, is sent to a foliation $\tilde{\mathscr{F}}_{\mu}^{u \mu}$ satisfying the corresponding properties. This means that $\left.h_{\mu}\right|_{N}$ preserves three fibrations: $\mathscr{F}_{\mu}^{u}, \Phi_{\mu}^{u} ; \mathscr{F}_{\mu}^{c}, \tilde{\mathscr{F}}_{\mu}^{c}$ and $\mathscr{F}_{\mu}^{\mu u}, \tilde{\mathscr{F}}_{\mu}^{u u}$, and $\mathscr{F}_{\mu}^{u u}, \tilde{F}_{\mu}^{u u}$, where $\mathscr{F}_{\mu}^{u u}$ is chosen arbitrarily, varying continuously with $\mu$. 
A direct computation shows that the angle between $\tilde{\mathscr{F}}_{\mu}^{u u}$ and $\tilde{W}_{\mu}^{c}$ is bounded away from zero, uniformly with $\mu$, and so the saturation of $\tilde{\mathscr{F}}_{\mu}^{\mu u}$ converges to the unique strong unstable foliation in $\tilde{W}_{\mu}^{u}\left(\tilde{q}_{1}\right)$ as $\mu_{1} \rightarrow 0$.

We extend $h_{\mu}$ to a neighbourhood of $W_{\mu}^{s}(p)$ and to a neighbourhood of $W_{\mu}^{c}\left(q_{1}\right)$ by conjugacy. It is easy to see that for each $\mu, h_{\mu}$ is continuous.

We now prove the continuity for each of $h_{\mu}$ on $\mu$.

(I) Continuity on $W_{\mu_{o}}^{s}(p), \mu_{o}=\left(\mu_{1}^{o}, \mu_{2}^{o}\right)$.

(a) if $\mu_{2}^{o} \neq 0$, then $h_{\mu}(x) / x^{\log \tilde{\alpha} / \log \alpha} \rightarrow k(\mu)$ along $\Gamma$ and we preserve $\mathscr{F}_{\mu}^{u}, \Phi_{\mu}^{u}$ in a fixed neighbourhood of $W_{\mu}^{u}(p)$. Hence, the continuity on $\mu$ is direct

(b) if $\mu_{o}=\left(\mu_{1}^{o}, 0\right)$ and we approach $\mu_{o}$ by values in the interior of the first quadrant, we proceed as follows.

Take $v_{\mu} \rightarrow v_{\mu_{o}}$ as $\mu \rightarrow \mu_{o}, v_{\mu_{o}} \in W_{\mu_{o}}^{s}(p) \backslash\{p\}$. Suppose $w_{\mu}=f^{n_{\mu}}=\left(v_{\mu}\right) \in R_{2} \backslash R_{4}$, i.e., $s_{o}(\mu) \leq w_{\mu} \leq s_{2}(\mu)$. Identifying $v_{\mu}, w_{\mu}$ with their second coordinate, we have

$$
\alpha^{-n_{\mu}} \boldsymbol{w}_{\mu}=v_{\mu} \rightarrow v_{\mu_{o}} \neq 0 \text {. }
$$

Remember that from Proposition 8,

$$
s_{o}(\mu)=c_{\mu}-\frac{3}{2} \mu_{2}^{2}, \quad s_{2}(\mu)=c_{\mu}+\mu_{1}^{2} \quad \text { and } \quad c_{\mu}=o\left(\mu_{2}\right)
$$

Also, $\tilde{s}_{o}(\mu)=k_{1}\left(\tilde{s}_{o}(\mu)\right) \cdot s_{o}(\mu)$ and $\tilde{s}_{2}(\mu)=k_{1}\left(\tilde{s}_{2}(\mu)\right) \cdot s_{2}(\mu)$ where $k_{1}\left(\tilde{s}_{i}(\mu)\right) \rightarrow$ $k\left(\mu_{o}\right), 1 \leq i \leq 2$, as $\mu \rightarrow \mu_{o}$. Moreover, $\tilde{c}_{\mu}=\tilde{s}_{o}(\mu)+\tilde{A}(\mu)\left(c_{\mu}-s_{o}(\mu)\right)^{\lambda}$, where $\tilde{A}(\mu)$ is a constant corresponding to $A(\mu)$ in $N$. As $s_{o}(\mu) \leq w_{\mu} \leq s_{2}(\mu)$ and $\tilde{s}_{o}(\mu) \leq \tilde{w}_{\mu} \leq$ $\tilde{s}_{2}(\mu)$ then

$$
\frac{\tilde{s}_{o}(\mu)}{s_{2}(\mu)} \leq \frac{\tilde{w}_{\mu}}{w_{\mu}} \leq \frac{\tilde{s}_{2}(\mu)}{s_{o}(\mu)} .
$$

But

$$
\frac{\tilde{s}_{o}(\mu)}{s_{2}(\mu)}=k_{1}(\tilde{s}(\mu)) \frac{s_{o}(\mu)}{s_{2}(\mu)}=k_{1}\left(\tilde{s}_{o}(\mu)\right) \frac{\left(c_{\mu}-\frac{3}{2} \mu_{2}^{2}\right)}{\left(c_{\mu}+\mu_{2}^{2}\right)} \rightarrow k(\mu)
$$

as $\mu \rightarrow \mu_{o}$.

Let us see that $\tilde{w}_{\mu} / w_{\mu} \rightarrow\left(\partial \tilde{\mu}_{2} / \partial \mu_{2}\right)\left(\mu_{1}, 0\right)$ as $\mu \rightarrow \mu_{o}$. Indeed,

$$
\begin{aligned}
\frac{\tilde{w}_{\mu}}{w_{\mu}} & =\frac{\tilde{\mu}_{2}+d_{\mu}\left(w_{\mu}+\mu_{2}\right)}{w_{\mu}}=\frac{\tilde{\mu}_{2} / \mu_{2} \cdot \mu_{2}+d_{\mu} w_{\mu}-d_{\mu} \mu_{2}}{w_{\mu}} \\
& =\left(\frac{\tilde{\mu}_{2}}{\mu_{2}}-d_{\mu}\right) \frac{\mu_{2}}{w_{\mu}}+d_{\mu}=\left(\frac{\tilde{\mu}_{2}}{\mu_{2}}-\frac{\partial \tilde{\mu}_{2}}{\partial \mu_{2}}\left(\mu_{1}, 0\right)\right) \frac{\mu_{2}}{w_{\mu}}+\left(\frac{\partial \tilde{\mu}_{2}}{\partial \mu_{2}}\left(\mu_{1}, 0\right)-d_{\mu}\right) \frac{\mu_{2}}{w_{\mu}}+d_{\mu}
\end{aligned}
$$

where $d_{\mu}=\left(\partial h_{\mu} / \partial y\right) /(\bar{y}), \bar{y} \in \Gamma$.

Now observe that calling $h\left(y, \mu_{2}\right)$ the conjugacy along $\Gamma$ for values $\mu=\left(0, \mu_{2}\right)$, a Taylor expansion yields

$$
\tilde{c}_{\mu}=\frac{\partial h}{\partial y}(\bar{y}) y+\frac{\partial h}{\partial \mu_{2}}\left(\bar{\mu}_{2}\right) \mu_{2} .
$$

Since $\tilde{c}_{\mu}=\tilde{\mu}_{2}+o\left(\tilde{\mu}_{2}^{2}\right)$ and $\left(\partial h / \partial \mu_{2}\right)\left(\bar{\mu}_{2}\right) \rightarrow 0$ as $\mu_{2} \rightarrow 0$, we get that $\partial \tilde{\mu}_{2} / \partial \mu_{2}=$ $\left(\partial h_{\mu} / \partial y\right)(0)$. So $d_{\mu} \rightarrow\left(\partial \tilde{\mu}_{2} / \partial \mu_{2}\right)\left(\mu_{1}, 0\right)$ and $\tilde{w}_{\mu} / w_{\mu} \rightarrow\left(\partial \tilde{\mu}_{2} / \partial \mu_{2}\right)\left(\mu_{1}, 0\right)$ since $\lim \sup _{\mu \rightarrow \mu_{o}}\left|\mu_{2} / w_{\mu}\right| \leq 1$.

Finally we see that if $w_{\mu} \alpha^{-n_{\mu}}=v_{\mu} \rightarrow v_{o}$ then $\tilde{w}_{\mu} \tilde{\alpha}^{-n_{\mu}}=\tilde{v}_{\mu} \rightarrow \tilde{v}_{o}$ :

$$
\frac{\tilde{w}_{\mu}}{w_{\mu}} \frac{\tilde{\alpha}^{-n_{\mu}}}{\alpha^{-n_{\mu}}}=\frac{\tilde{w}_{\mu}}{w_{\mu}}\left(\frac{\tilde{\alpha}}{\alpha}\right)^{-n_{\mu}} \text {. }
$$


We have just verified that $\tilde{w}_{\mu} / w_{\mu} \rightarrow\left(\partial \tilde{\mu}_{2} / \partial \mu_{2}\right)\left(\mu_{1}, 0\right)$; and

$$
\begin{aligned}
\frac{\tilde{s}_{2}(\mu)}{s_{o}(\mu)} & =k_{1}\left(\tilde{s}_{2}(\mu)\right) \frac{s_{2}(\mu)}{s_{o}(\mu)} \\
& =k_{1}\left(\tilde{s}_{2}(\mu)\right) \frac{c_{\mu}+\mu_{2}^{2}}{c_{\mu}-\frac{3}{2} \mu_{2}^{2}} \rightarrow k(\mu)
\end{aligned}
$$

as $\mu \rightarrow \mu_{o}$.

Now $\tilde{v}_{\mu}=\tilde{\alpha}^{-n_{\mu}} \tilde{w}_{\mu}=\alpha^{-n_{\mu}} \tilde{w}_{\mu}$ because $a=\tilde{\alpha}$, due to our reparametrization. Thus $\tilde{v}_{\mu}=\alpha^{-n_{\mu}} \tilde{w}_{\mu}=\left(\alpha^{-n_{\mu}} \tilde{w}_{\mu} / w_{\mu}\right) \cdot w_{\mu}$ which converges to $k\left(\mu_{o}\right) \cdot v_{\mu_{o}}$ as $\mu \rightarrow \mu_{o}$. And we have proved continuity on $W_{\mu_{0}}^{s}(p)$.

(b) If $\mu_{o}=\left(\mu_{1}^{o}, 0\right)$ and we approach $\mu_{o}$ by values in the fourth quadrant, take $v_{\mu} \rightarrow v_{\mu_{o}}$ as $\mu \rightarrow \mu_{o}, v_{\mu_{o}} \in W_{\mu_{o}}^{s}(p) \backslash\{p\}$. Note that in this case $\alpha_{\mu}$ and $\tilde{\alpha}_{\mu}$ may be different.

Let $w_{\mu}=f^{n_{\mu}}\left(v_{\mu}\right) \in N$. Identifying $v_{\mu}, w_{\mu}$ with their second coordinate we have:

$$
\alpha^{n} v_{\mu}=w_{\mu} \text {. }
$$

If $w_{\mu} \geq s_{2}(\mu), h_{\mu}\left(w_{\mu}\right)=k(\mu) w_{\mu}^{\log \tilde{\alpha} / \log \alpha}$ and the result easily follows. Otherwise let $\lim \inf _{\mu \rightarrow \mu_{o}}\left|w_{\mu} / \mu_{2}\right| \geq 1$.

We claim that $\mu_{2} n_{\mu} \rightarrow 0$ as $\mu \rightarrow \mu_{o}$. In fact, lim inf $\mu_{\mu \rightarrow \mu_{o}}\left|w_{\mu} / \mu_{2}\right| \geq 1$ together with $\left(w_{\mu} / \mu_{2}\right) \mu_{2} \cdot \alpha^{-n_{\mu}} \rightarrow v_{\mu_{o}}$ imply $\left|\mu_{2}\right| \alpha^{-n_{\mu}}<\delta$ for some constant $\delta>0$. Taking logarithms the inequality turns into

$$
\log \left|\mu_{2}\right|-n_{\mu} \log \alpha<\log \delta
$$

and multiplying by $\left|\mu_{2}\right|$ we get

$$
\left|\mu_{2}\right| \log \left|\mu_{2}\right|-\left|\mu_{2}\right| n_{\mu} \log \alpha<\left|\mu_{2}\right| \log \delta .
$$

So $\left|\mu_{2}\right| n_{\mu} \rightarrow 0$ and the claim is proved.

Also $\tilde{\alpha} / \alpha=1+o\left(\mu_{2}\right)$ because $\tilde{\alpha}=\alpha$ at the axis $\mu_{2}=0$. So

$$
\left(\frac{\tilde{\alpha}}{\alpha}\right)^{-n_{\mu}}=\left(1+o\left(\mu_{2}\right)\right)^{-n_{\mu}}=\left[\left(1+o\left(\mu_{2}\right)\right)^{1 / \mu_{2}}\right]^{-\mu_{2} n_{\mu} \rightarrow 1}
$$

as $\mu_{2} \rightarrow \mu_{o}$.

Thus $\left(\tilde{w}_{\mu} / w_{\mu}\right)(\tilde{\alpha} / \alpha)^{-n_{\mu}} \rightarrow\left(\partial \tilde{\mu}_{2} / \partial \mu_{2}\right)(\mu, 0)$ as $\mu \rightarrow \mu_{o}$ and this concludes the proof of the continuity on $\mu$ in a neighbourhood of $W_{\mu_{o}}^{s}(p)$.

(II) Continuity on $W_{\mu_{s}}^{u}\left(q_{1}\right)$.

Take $v_{\mu} \rightarrow v_{o}$ as $\mu \rightarrow \mu_{o}$. The interesting cases are when $\mu_{o}=\left(0, \mu_{2}^{o}\right)$ and $v_{o} \epsilon$ $W_{\mu_{o}}^{u}(q)$.

(II.a) We first consider $\mu=\left(\mu_{1}, \mu_{2}\right) \rightarrow \mu_{o}, \mu_{2} \leq 0$.

In this case $S_{\mu_{1}}=S_{\mu_{2}}=\Phi, \lambda \equiv 1$. As we preserve $\mathscr{F}_{\mu}^{c}, \tilde{F}_{\mu}^{c}$ and $\mathscr{F}_{\mu}^{u \mu}, \tilde{\mathscr{F}}_{\mu}^{u u}$ the convergence is direct when $\mu_{1}>0$. Also, when $\mu_{1} \leq 0$, we preserve $\mathscr{F}_{\mu}^{c}, \tilde{\mathscr{F}}_{\mu}^{c}$ and the continuity follows.

If $\mu_{2}>0$ then for $\mu_{1}$ small enough we have $S_{\mu_{2}} \supseteq S_{\mu_{1}}$. Note that as $\mu_{2}>0$, the saturation of $S_{\mu_{2}}$ by $f_{\mu}$ covers a fixed neighbourhood of $q_{1}$. Then the continuity follows because we are preserving $\mathscr{F}_{4}, \tilde{\mathscr{F}}_{4}$ and $\mathscr{F}_{\mu}^{c}, \tilde{\mathscr{F}}_{\mu}^{c}$ and $\mathscr{F}_{4}$ converges to $\mathscr{F}^{u u}$.

When $\mu_{2}^{o}=0$ we consider the values of $\mu$ such that
(1) $S_{\mu_{2}}=\Phi$,
(2) $S_{\mu_{2}} \supseteq S_{\mu_{1}}$ and
(3) $S_{\mu_{2}} \supseteq S_{\mu_{2}}$. 
We have just analysed above the first two possibilities. So it is enough to take $w_{\mu} \rightarrow w_{o}$ as $\mu_{\mu} \rightarrow 0, S_{\mu_{1}} \supseteq S_{\mu_{2}}$.

Let us make a few observations about the saturation of our fibrations.

Due to the choice of $s_{o}, \tilde{s}_{\mathrm{o}}, \mathscr{F}_{\mu}^{u u}$ and $\tilde{\mathscr{F}}_{\mu}^{u u}$, the angle between $\mathscr{F}^{c}$ and $\mathscr{F}^{u u}$ in $S_{\mu_{1}}$ has at'least the order of $\mu_{2}$. As the width of $S_{\mu_{2}}$ has the order of $\mu_{2}^{2}$, we conclude, by a direct computation, that $\mathscr{F}_{\mu}^{u u}$ restricted to $S_{\mu_{1}} \backslash S_{\mu_{2}}$, when iterated, converges to a fibration whose fibers are contained in those of the strong foliation in $W_{o}^{u}\left(q_{1}\right)$. Analogously for $\tilde{\mathscr{F}}_{\mu}^{\text {uu }}$.

Reasoning in the same way we can see that either the saturation of $S_{\mu_{2}}$ by $f_{\mu}$ collapses to $W_{\mu}^{c}\left(q_{1}\right)$ or, when iterated, $\mathscr{F}_{4}$ converges to the strong foliation in $W_{o}^{u}\left(q_{1}\right)$. This means that the foliation $\mathscr{F}_{5}=\mathscr{F}_{5}(\mu)$ of $S_{\mu_{1}}$ given by:

(a) outside $S_{\mu_{2}}$ it coincides with $\mathscr{F}_{\mu}^{\mu u}$

(b) inside $S_{\mu_{2}}$ it coincides with $\mathscr{F}_{\mu}^{\mu}$,

when iterated, converges to the strong unstable foliation in $W_{o}^{u}\left(q_{1}\right)$.

Similarly we define $\tilde{\mathscr{F}}_{5}$ in $\tilde{S}_{\mu_{1}}$, just replacing $\mathscr{F}_{\mu}^{u}$ by $\Phi_{\mu}^{u}$; when iterated it also converges to the strong unstable foliation in $W_{o}^{u}\left(\tilde{q}_{1}\right)$.

Observe that the homeomorphism in $\partial S_{\mu_{2}}$ is given by $\left.h_{\mu}\right|_{w^{c}}$ projected through $\mathscr{F}_{4}$, $\tilde{F}_{4}$. As $S_{\mu_{2}}$ shrinks, it converges to $\left.h_{\mu}\right|_{w_{\mu}^{c}}$. So, asymptotically with $\mu$, the saturation of $\mathscr{F}_{5}$ (resp. $\tilde{\mathscr{F}}_{5}$ ) is the strong unstable foliation in $W_{o}^{u}\left(q_{1}\right)$ (resp. $W_{o}^{u}\left(\tilde{q}_{1}\right)$ ).

Let us now return to our sequence $w_{\mu} \rightarrow w_{o} \in W^{u}\left(q_{1}\right), S_{\mu_{1}} \supseteq S_{\mu_{2}}$.

If $\left\{w_{\mu}\right\} \subset S_{\mu_{2}}$, since $h_{\mu}$ preserves $\mathscr{F}^{c}, \tilde{F}^{c}$ and $\mathscr{F}_{5}, \tilde{F}_{5}$ then $h_{\mu}\left(w_{\mu}\right) \rightarrow h_{o}\left(w_{o}\right)$ as $\mu \rightarrow 0$. Otherwise $\left\{w_{\mu}\right\} \subseteq S_{\mu_{1}} \backslash S_{\mu_{2}}$.

On account of (6.1) and the preservation of $\mathscr{F}_{5}, \tilde{\mathscr{F}}_{5}, h_{\mu}\left(w_{\mu}\right) \rightarrow h_{o}\left(w_{o}\right)$ as $\mu \rightarrow 0$ too. (II.b) We consider $\mu=\left(\mu_{1}, \mu_{2}\right) \rightarrow \mu_{o}, \mu_{1} \leq 0$.

When we approach $\mu_{o}$ by values $\mu=\left(\mu_{1}, \mu_{2}\right)$ with $\mu_{2} \leq 0$, we are preserving $\mathscr{F}^{u}$, $\Phi^{\mu}$ and $\mathscr{F}^{c}, \tilde{\mathscr{F}}^{c}$. This grants continuity.

So we deal with $\mu=\left(\mu_{1}, \mu_{2}\right), \mu_{2}>0$.

In this case it is enough to consider sequences $v_{\mu} \rightarrow v_{o} \in W^{u u}\left(q_{1}\right)$ for which $w_{\mu}=f_{\mu}^{-n_{k}}\left(v_{\mu}\right) \in f_{\mu}^{j_{o}}\left(R_{4} \cap R_{1}\right)$, where $f_{\mu}^{j_{o}}\left(R_{4} \cap R_{1}\right)$ is contained in a domain of partial linearization around $q_{1}$.

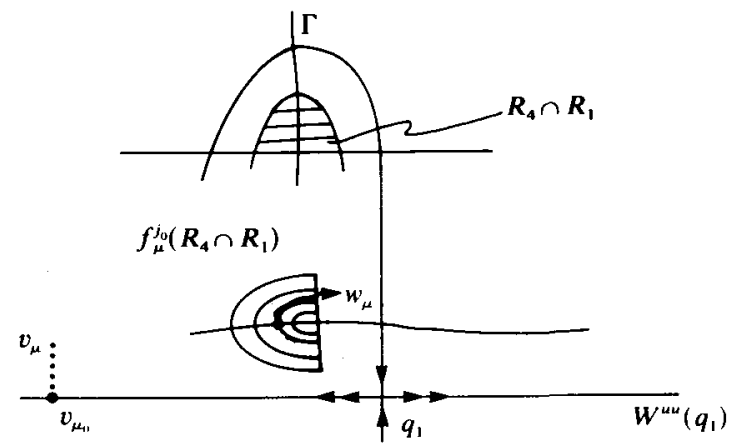

Identifying all points with their second coordinate in the partially linearizing coordinates,

$$
v_{\mu}=w_{\mu} \prod_{j=0}^{n_{k}-1} b_{\mu}\left(f_{\mu}^{j}\left(w_{\mu}\right)\right) \rightarrow v_{\mu_{o}} \neq 0,
$$


which implies

$$
v_{\mu}^{\lambda}=w_{\mu}^{\lambda} \prod_{j=0}^{n_{k}-1} b_{\mu}^{\lambda}\left(f_{\mu}^{j}\left(w_{\mu}\right)\right) \rightarrow v_{\mu_{o}} .
$$

We have to prove that

$$
\tilde{w}_{\mu} \prod_{j=0}^{n_{k}-1} \tilde{b}_{\mu}\left(\tilde{f}_{\mu}^{j}\left(\tilde{w}_{\mu}\right)\right) \rightarrow \tilde{v}_{\mu_{o}}
$$

Observe that as

$$
w_{\mu}=f_{\mu}^{j_{\mu}}\left(s_{o}(\mu)\right)+\left(\omega_{\mu}-f_{\mu}^{j_{o}}\left(s_{o}(\mu)\right)\right)
$$

then

$$
\tilde{w}_{\mu}=A f_{\mu}^{j_{\mu}}\left(s_{o}(\mu)\right)^{\lambda}+k_{2}\left(w_{\mu}\right)\left(w_{\mu}-f_{\mu}^{j_{\mu}}\left(s_{o}(\mu)\right)\right) .
$$

Since $f_{\mu}^{j_{o}}\left(s_{o}(\mu)\right)=o\left(\mu_{2}\right)$ and $w_{\mu}-f_{\mu}^{j_{o}}\left(s_{o}(\mu)\right)=o\left(\mu_{2}^{2}\right)$ on account of the choice of $s_{o}(\mu)$,

$$
\begin{aligned}
\frac{\tilde{w}_{\mu}}{w_{\mu}^{\lambda}} & =\frac{\left(f_{\mu}^{j_{o}}\left(s_{o}(\mu)\right)\right)^{\lambda}\left[A+k_{2}\left(w_{\mu}\right)\left(\frac{w_{\mu}-f_{\mu}^{j_{o}}\left(s_{o}(\mu)\right)}{f_{\mu}^{j_{o}}\left(s_{o}(\mu)\right)^{\lambda}}\right)\right]}{\left(f_{\mu}^{j_{o}}\left(s_{o}(\mu)\right)\right)^{\lambda}\left(1+\frac{w_{\mu}-f_{\mu}^{j_{o}}\left(s_{o}(\mu)\right)}{f_{\mu}^{j_{o}}\left(s_{o}(m)\right)}\right)^{\lambda}} \\
& =\frac{A+o\left(\mu_{2}^{2-\lambda}\right)}{\left[1+o\left(\mu_{2}\right)\right]^{\lambda}} \rightarrow A \text { as } \mu \rightarrow \mu_{o} .
\end{aligned}
$$

Finally,

$$
\begin{aligned}
\tilde{v}_{\mu} & =\tilde{w}_{\mu} \prod_{j=0}^{n_{k}-1} \tilde{b}_{\mu}\left(\tilde{f}_{\mu}^{j}\left(w_{\mu}\right)\right) \\
& =\frac{\tilde{w}_{\mu}}{w_{\mu}^{\lambda}} w_{\mu}^{\lambda} \prod_{j=0}^{n_{k}-1} b_{\mu}^{\lambda}\left(f_{\mu}^{j}\left(w_{\mu}\right)\right) \prod_{j=0}^{n_{k}-1} \frac{\tilde{b}_{\mu}\left(\tilde{f}_{\mu}^{j}\left(\tilde{w}_{\mu}\right)\right)}{b_{\mu}^{\lambda}\left(f_{\mu}^{j}\left(w_{\mu}\right)\right)} .
\end{aligned}
$$

By Proposition 7, we have

$$
\prod_{j=0}^{n_{k}-1} \frac{\tilde{b}_{\mu}\left(\tilde{f}_{\mu}^{j}\left(\tilde{w}_{\mu}\right)\right)}{b_{\mu}^{\lambda}\left(f_{\mu}^{j}\left(w_{\mu}\right)\right)} \rightarrow 1 \quad \text { as } \mu \rightarrow \mu_{o},
$$

and using the limits calculated above, we get

$$
\tilde{v}_{\mu} \rightarrow A\left(\mu_{o}\right) v_{\mu_{o}} \text { as } \mu \rightarrow \mu_{o}
$$

\section{REFERENCES}

[1] J. Beloqui. Modulus of stability for vector fields on 3-manifolds. J. Diff. Equations 65 (1986), 374-395.

[2] R. Bogdanov. Deformation verselle d'un point singulier d'un champ de vecteurs sur le plan dans le cas de valeurs propres nulles. Petrovski Seminar 2 (1976).

[3] P. Bonckaert, F. Dumortier \& S. Van Strien. Singularities in $\mathbf{R}^{2}$ determined by their homogeneous part. To appear.

[4] A. Chenciner. Bifurcations des points fixes elliptiques. Publ. Math. I.H.E.S. 61 (1985), 67-127.

[5] W. de Melo. Moduli of stability of two dimensional diffeomorphisms. Topology 19 (1980), 9-21.

[6] W. de Melo \& S. Van Strien. Diffeomorphisms on surfaces with a finite number of moduli. Ergod. Th. \& Dynam. Syst. 7 (1987), 415-462.

[7] J. Gheiner. On codimension-two bifurcations of diffeomorphisms. Thesis, IMPA. 
[8] J. Palis. A differential invariant of topological conjugacies and moduli of stability. Astérisque $\mathbf{5 1}$ (1978), 335-346.

[9] J. Palis \& S. Smale. Structural Stability Theorems, Global Analysis. vol. XIV, Amer. Math. Soc.: 1970. 223-231.

[10] S. Newhouse \& J. Palis. Cycles and bifurcation theory. Astérisque 31 (1976), 43-140.

[11] S. Newhouse, J. Palis \& F. Takens. Bifurcations and stability of families of diffeomorphisms. Publ. Math. I.H.E.S. 57 (1983), 5-72.

[12] S. Sternberg. On the structure of local homeomorphisms of Euclidean $n$-space, II. Amer. J. of Math. 80 (1958), 623-631.

[13] F. Takens. Partially hyperbolic fixed points. Topology 10 (1971), 133-148.

[14] F. Takens. Forced oscillations and bifurcations. Commun. Math. Institute, Rijksuniversiteit Utrecht (1974).

[15] S. J. Van Strien. One parameter families of vector fields, bifurcations near saddle-connections. (1982) Thesis, Utrecht. 